VIERTELJAHRSCHRIFT FÜR SOZIAL- UND WIRTSCHAFTSGESCHICHTE 106, 2019/1, 29-66

DOI 10.25162/VSWG-2019-0003

ANGELA HUANG / ALEXANDRA SAPOZNIK

\title{
Fremdes Geld
}

Auswärtige Kapitalbeziehungen des Braunschweiger Rentenmarktes im 15. und 16. Jahrhundert

\section{Foreign Capital}

Brunswick's Annuity Market and its External Relations in the $15^{\text {th }}$ and $16^{\text {th }}$ Centuries

ABSTRACT: A number of studies have looked into local annuity markets, mainly focusing on local conditions. This article explores capital networks for urban debt by analyzing the external relations of the Brunswick annuity market in the $15^{\text {th }}$ and $16^{\text {th }}$ centuries. Our study is based on an examination of data on foreign buyers of Brunswick (Braunschweig) annuities from the unpublished 'Weddeschatbücher' that registered sales of redeemable annuities. The analysis takes into account different groups of buyers and reveals spatial patterns corresponding with those of commodity trade and political cooperation within and beyond the region. A closer look at the chronology of sales indicates that political crises were the main cause of urban debt and foreign investment. In particular, the Reformation led to structural changes to the supply of foreign capital. Although interest rates provided economic incentives for foreign buyers, political support was the predominant reason for both individuals and other communes to supply capital in times of need. Keywords: Annuity markets, capital networks, urban debt JEL-Codes: $\mathrm{N}_{23}, \mathrm{~N}_{93}, \mathrm{O}_{16}, \mathrm{P}_{43}$

\section{Einleitung}

Das öffentliche Haushaltswesen und hier insbesondere die Kreditnahme haben sich in der Wirtschaftsgeschichte in der jüngeren Vergangenheit erneuter Beliebtheit erfreut wenig überraschend vor dem Hintergrund von Banken-, Finanz- und Schuldenkrisen im frühen 21. Jahrhundert. Bereits das Spätmittelalter war dabei eine Zeit ausgedehnter Kreditwirtschaft, der Rentenkauf wohl das bedeutendste Kreditinstrument nördlich der Alpen. Seit dem späten 14. Jahrhundert verkauften die meisten deutschen Städte regelmäßig Leib-, aber vor allem Erbrenten. ${ }^{1}$ Rentenanleihen machten oftmals ein Viertel oder mehr der jährlichen Einnahmen und Ausgaben aus. ${ }^{2}$

1 Leibrentenverträge berechtigten den Rentenkäufer zu jährlichen Rentenzahlungen für die Lebensdauer der im Vertrag genannten Begünstigten und sind im Normalfall nicht kündbar. Erbrenten sind mit dem Spätmittelalter üblicherweise nicht nur vererbbar, sondern auch frei übertragbar und mindestens durch den Rentenverkäufer gegen Rückzahlung des vollen Kapitals kündbar.

2 In Auswahl: Isenmann (2014), S. 949-957; Gilomen (1984), S. 1, 49-53; Cremer (1937), S. 105-107; Fuhrmann (2016), S. 11 f., 16-18. 
Dabei stellen die deutschen Städte, die im Folgenden am Braunschweiger Beispiel behandelt werden, eine eigene Gruppe in der Diskussion um Märkte für öffentliche Anleihen in der Vormoderne dar. Dies gilt insbesondere für die Freien und Reichsstädte sowie für die de facto autonomen Hansestädte mit ihren weitreichenden Selbstverwaltungsrechten. Im Gegensatz zur unabhängigen Finanzpolitik der deutschen Städte sind die Rentenverkäufe der niederländischen Städte in die territorialstaatliche Finanzpolitik eingebunden. Die niederländischen Städte waren oftmals stellvertretende Kreditnehmer ihrer Fürsten und leisteten derart einen Beitrag zum Staatenbildungsprozess der burgundischen Niederlande, ${ }^{3}$ während die deutschen Städte Renten vorrangig für ihre eigenen politischen und wirtschaftlichen Ziele verkauften. ${ }^{4}$

In der Auseinandersetzung mit dem Rentenkauf der deutschen Städte wurden bislang auswärtige Rentenkäufer zumeist nur am Rande thematisiert, ${ }^{5}$ obwohl die überlokale Verfügbarkeit und Mobilität von Kapital eine wichtige Dimension in die Diskussion um Haushaltswesen und Kreditmärkte einbringt. Vor allem Kriege und Fehden, welche die städtische Wirtschaft störten und städtische Unabhängigkeit bedrohten, erforderten die kurzfristige Beschaffung größerer Summen, was für die meisten Städte nicht ohne auswärtiges Kapital zu leisten war. ${ }^{6}$ Inwieweit diese Städte grundsätzlich oder zur Sicherung ihrer wirtschaftlichen und politischen Interessen auf auswärtiges Kapital angewiesen waren und welcher Art die Kreditnetzwerke zur Bereitstellung von Kapital waren, wurde bislang noch nicht gezielt bearbeitet. Der vorliegende Beitrag möchte mit einer Untersuchung der auswärtigen Kapitalbeziehungen des Braunschweiger Rentenmarktes zu dieser Frage einen Beitrag leisten.

\section{Forschungsstand und Quellen}

Bereits die vorliegenden Fallstudien zu einzelnen Rentenmärkten weisen auf einen grundsätzlich auch überlokalen Charakter des städtischen Rentenkaufes hin. Rentenverkäufe an Auswärtige sind für jeden untersuchten Rentenmarkt zu beobachten. Die Anlagegeschäfte Lübecker Bürger in Mecklenburg im 13. Jahrhundert sind ein frühes Beispiel dafür, dass überlokale Rentengeschäfte mit der Etablierung der städtischen Geldrente einsetzen und regelmäßig belegt sind.?

3 Beispielhaft Fryde/Fryde (1963), S. 492-507, 527, 540; Zuijderduijn (2009); Lambert (2015); Bosch (2015); Haemers (2015).

4 Fryde/Fryde (1963), S. 527, 542.

5 Zu Auswärtigen im städtischem Haushalt bzw. Rentenkauf kurz Isenmann (2014), S. 546 f. Wenige Arbeiten befassen sich gezielter mit Auswärtigen: Gilomen (2003), S. 180-185; Gilomen (2007), S. 25-74; Gilomen (2018), v. a.S. 78-96; Rothmann (2007), S. 183-238; Fuhrmann (2007), S. 139-167; Fuhrmann (2016), S. 169-215. Zur Mobilität von Kapital im Heiligen Römischen Reich: Chilosi/Schulze/Volckart (2018).

6 Fouquet (1989); Fuhrmann (2016), S. 26-30; Rothmann (2007), S. 183 f., 192, 201; Albers (1939), S. 24 f.; Fuhrmann (2003), S. 4 f., 7, 11; Gilomen (2003), S. 166; Hefenbrock (1929), S. 32 f., 44 f.; Knipping (1894), S. 347 f., 351 f., $355-357,360,363-367$.

7 Für Wismar sind Rentenkäufe in den Jahren 1300, 1327 und 1328, Rentenzahlungen an einen Lübecker für 
Die bisher geleisteten Untersuchungen zu lokalen Rentenmärkten nennen dabei unterschiedliche Anteile von Auswärtigen für die einzelnen Städte. Die wirtschaftsstarke Stadt Köln etwa konnte im 14. und 15. Jahrhundert weitestgehend auf das Kapital der eigenen Bürgerschaft zurückgreifen und hat nur notfalls an Auswärtige verkauft, wobei man sich um baldige Rückzahlung bemühte. Insbesondere äußere Konflikte erforderten allerdings auch Kapitalaufnahmen von Auswärtigen, so im letzten Drittel des 14. Jahrhundert von Bürgern der Städte Mainz, Frankfurt und in geringerem Maße Lübeck. Zwischen 1418 und 1431 kamen um die 50 Prozent der Einnahmen Kölns aus Erbrentenverkäufen; ca. 20 Prozent der aufgenommenen Kapitalien kamen von auswärts, namentlich Frankfurt und Mainz. ${ }^{8}$ Der Stadtrentenverkauf soll auch in Breslau im 14. und frühen 15. Jahrhundert vor allem innerstädtisch stattgefunden haben. Im Zeitraum 1358 bis 1387 entfielen dann allerdings fast 40 Prozent der verkauften Leibrenten allein auf Thorn, mit dem Breslau die engsten Handelsbeziehungen unterhielt. Weitere Rentenkäufe durch Bürger von Posen, Kulm, Danzig, Krakau, Nürnberg, Soest und Köln werden mit dem sich ausdehnenden Breslauer Handel in Zusammenhang gebracht. ${ }^{9}$ In Bremen erscheinen auswärtige Rentenkäufer erst im 15. Jahrhundert, dann zunächst aus Lüneburg, Stade, Lübeck, Buxtehude, Wismar und Deventer. In den folgenden Jahrhunderten blieben Auswärtige präsent auf dem Bremer Rentenmarkt, mit wechselnder Herkunft des Kapitals. ${ }^{10}$ Stellten Lübecker Kapitalien 1426 bis 1453 knapp die Hälfte der Hamburger Kapitalaufnahmen, hatten Auswärtige und v. a. Lübecker hier in der zweiten Hälfte des 15. Jahrhunderts nur einen Anteil von 17 Prozent. Seit den $1550 e r n$ spielten Lübecker Gelder keine Rolle mehr, was mit einer größeren Eigenständigkeit der hamburgischen Politik erklärt wird. ${ }^{11}$ In Kiel waren auswärtige Anleger 1488 bis 1560 eine Ausnahme; für Krempe ist nur ein Anteil von knapp 14 Prozent belegt. ${ }^{12}$ In Schwäbisch Hall wurden Gelder bis 1540 innerhalb der Stadt aufgebracht. In der Folgezeit wurden Kapitalien auch von benachbarten Städten, wie Augsburg, Ulm und Heilbronn, und von Adligen aufgenommen, wobei weiterhin die meisten Renten in Schwäbisch Hall verkauft wurden. ${ }^{13}$ Während im 14. Jahrhundert Mainz und in der Folgezeit Speyer und Nürnberg wichtige Kapitalgeber von Rothenburg ob der Tauber waren, kamen ab 1428 Rentenempfänger vor allem aus der Stadt selbst. ${ }^{14}$ Auch in München herrschten Anlagen Einheimischer vor. Soweit auswärtige Geldgeber belegt sind, handelt es sich vorrangig um Augsburger. Umgekehrt kauften Münchener vornehmlich in Augsburg Renten. ${ }^{15}$ Während Basel den überwiegenden Teil seiner Renten an eigene Bürger und Hintersassen verkaufte, waren Zürich und Bern stark auf auswärtiges und vor allem Ba-

1326/27 bis zuletzt 1333/34 belegt; für Rostock sind Rentenzahlungen im Lübecker Niederstadtbuch 1334 bezeugt, Rentenverkäufe 1362, 1371 und 1377. Hefenbrock (1929), S. 27, 32 f., 57-60, 65, 81 (Tabelle), $84,86$. 
seler Kapital angewiesen. Berner selbst trugen etwa in der zweiten Hälfte des 15. Jahrhunderts nur zwischen 2,6 und 5 Prozent der Rentenschuld, Basel den Löwenanteil von 32,6-43,5 Prozent und das weit entfernte Nürnberg immerhin um die 10-15 Prozent der Rentenschuld. Zürich zahlte in den 1440ern 17,8 Prozent der Rentensummen in der eigenen Stadt aus, weitere 17,4 Prozent an Baseler und zehn Prozent an Bürger aus Aarau. Die restliche Rentenschuld verteilt sich auf 26 weitere Märkte. ${ }^{16}$

Die vorliegenden Fallstudien erlauben einige allgemeine Aussagen zur Rolle Auswärtiger auf den Rentenmärkten für städtische Schuld in den Städten des spätmittelalterlichen Reichs. Bei wechselnden Anteilen Auswärtiger wird der innerstädtische Verkauf von Stadtrenten als Normalfall angesehen; nach Möglichkeit stellten die eigenen Bürger die Gläubiger der Stadt. Hohe Anteile von Auswärtigen ergaben sich aus kurzfristig erhöhtem Geldbedarf der Städte, wie bereits erwähnt v. a. im Zusammenhang mit äußeren Konflikten. Kapitalien wurden aber auch für Bürgschaften der Städte gegenüber Dritten aufgenommen. ${ }^{17}$ War es nötig, dass man bei Auswärtigen Kapital lieh, so bemühten sich die Städte, diese Kredite schnell zu tilgen oder bald auf Einheimische umzuschichten. Fanden Kapitalaufnahmen von außen statt, so waren diese durch wirtschaftliche und politische Beziehungen geprägt. Enge Kapitalbeziehungen bestanden zwischen kleineren Städtegruppen, die oft über den Handel bereits dauerhafte Wirtschaftsbeziehungen etabliert hatten. Im oberdeutschen Raum und in der Schweiz spielte der Adel tendenziell eine größere Rolle.

Als politischer Grund gegen auswärtige Stadtrentenkäufer wird von der Forschung die Gefahr der Abhängigkeit nach außen genannt. Wirtschaftliche Gründe gegen Kapitalaufnahmen von außen bestanden durch höhere Transaktionskosten für die verkaufende Stadt. Verkauf außerhalb der Stadt bedeutete zudem Abfluss von Edelmetall, Kosten für Vermittlung sowie erhöhte Kosten für Administration und Kontrolle (handling costs) und die damit verbundenen Risiken. ${ }^{18}$ Stadtrentenverkäufe nach außen werden somit insgesamt als ein notwendiges Übel bewertet, mit negativen politischen und fiskalischen Folgen.

Und doch hatte jede Stadt früher oder später Kapitalbedarf, der nicht innerstädtisch zu decken war. Geldaufnahmen von außen waren keine vermeidbare Option, sondern fester Bestandteil des städtischen Finanzwesens. Für das Spätmittelalter wurde dabei insgesamt ein großes Geldangebot am Kapitalmarkt und insbesondere für langfristige Anlagen festgestellt. ${ }^{19}$ Kapitalstarke Bürger von Städten wie Nürnberg und Basel suchten nach auswärtigen Anlagemöglichkeiten. ${ }^{20}$ Vor allem seit dem 16. Jahrhundert nahm die Mobilität von Kapital zwischen den Städten des Heiligen Römischen Reiches deutlich zu. Während der Schwerpunkt der Kapitalbeziehungen in einem Radius von $200 \mathrm{~km}$ lag, lassen sich insgesamt größere Reichweiten und höhere Summen beobach- 
ten. ${ }^{21}$ Eine stärkere Beteiligung von Auswärtigen im 16. Jahrhundert soll sich dabei aus allgemein besseren Bedingungen für überlokale Kapitalbeziehungen ergeben haben. ${ }^{22}$ Als mögliche Gründe für die positive Entwicklung institutioneller Rahmenbedingungen im Heiligen Römischen Reich wurden die städtischen Gestaltungsmöglichkeiten im Rechtswesen, die fortgesetzte politische Kooperation der Städte und der friedliche Wettbewerb um auswärtiges Kapital benannt. ${ }^{23}$ Das aktivierbare Kapitalangebot ergab sich aus den städtischen Vernetzungen in der Region und darüber hinaus, konstituiert durch politische Beziehungsnetzwerke, Handelsbeziehungen und persönlich-familiäre Beziehungen. ${ }^{24}$ Messen, regionale Kapitalmärkte und Geldhändler spielten in der Vermittlung von Kapitalüberschüssen v. a. von Einzelpersonen eine wichtige Rolle. Kirchliche Einrichtungen und Ratskollegien wurden dahingegen bei Geldbedarf mitunter gezielt angefragt. ${ }^{25}$ Engere Kreditbeziehungen sollen etwa zwischen den Reichsstädten bestanden haben; ähnliche Netzwerke werden für die Städte am Oberrhein, am Mittelrhein und in Oberdeutschland angenommen. ${ }^{26}$ Die Außenbeziehungen der Städte im Rentenkauf erfüllten im Finanzbereich einen wichtigen Zweck, da sie der Stadt in Zeiten erhöhten Kapitalbedarfs den notwendigen Kredit zur Verfügung stellen konnten.

Vor diesem Hintergrund nimmt die vorliegende Untersuchung die auswärtigen Kreditbeziehungen des Braunschweiger Rentenmarktes in den Blick. ${ }^{27}$ Braunschweig bietet sich für eine Fallstudie aufgrund der guten Materiallage an. Diese erlaubt es, seit dem späteren 14. Jahrhundert die Rentenverkäufe der Stadt anhand der Amtsbuchserie der noch unveröffentlichten ,Weddeschatbücher' nachzuverfolgen. ${ }^{28}$ Die Bücher setzen in den $1360 e r$ Jahren ein. In ihnen sind wohl ein Großteil, wenn nicht alle Rentenverkäufe der Stadt eingetragen, ebenso zahlreiche Konversionen und Rückkäufe von Renten. Die Laufzeit der,Weddeschatbücher' beginnt nur kurz nach der Entwicklung einer gemeinsamen Stadtverwaltung und eines selbstverwaltenden Gemeinwesens in der Mitte des 14. Jahrhunderts und reicht bis zum Ende der städtischen Freiheit im 17. Jahrhundert. ${ }^{29}$

Für die vorliegende Fragestellung haben wir sechs der insgesamt zwölf,Weddeschatbücher' für den Zeitraum 1361 bis 1599 ausgewertet. Wir untersuchen den Braunschwei-

Chilosi/Schulz/Volckart (2018), S. 651-656.

Albers (1939), S. 50.

Chilosi/Schulz/Volckart (2018), S. 662-665.

Isenmann (2014), S. 546-547; Rothmann (2007), S. 189.

Albers (1939), S. 43 f.; Rothmann (1998), S. 423-478; Rothmann (2007); Fuhrmann (2016), S. 20 f.; Gilomen (2003), S. 182; Gilomen (2018), S. 79.

Fuhrmann (2003), S. 16.

Zum Braunschweiger Rentenmarkt liegen bereits Untersuchungen vor, die sich aber auf den lokalen Markt beschränken: Mack (1889); Bohmbach (1969).

Stadtarchiv Braunschweig, B I 12, Weddeschatbücher, 12 Bde., hier Bände 1-6. Leibrentenbücher liegen ebenfalls vor (B I 11, 6 Bde.), konnten aber wegen der Vergleichbarkeit der Beobachtungen, der vorrangigen Natur von Leibrenten als innerstädtische Vorsorgeinstrumente und wegen des Umfangs des Materials nicht berücksichtigt werden.

Die fünf Teilstädte bzw. Weichbilde Alstadt, Hagen, Neustadt, Altenwik und Sack schlossen sich seit dem späten 13. Jh. zur Gemeinen Stadt Braunschweig zusammen mit einer gemeinsamen Finanzverwaltung neben denen der Teilstädte. Mitte des 14. Jh.s kontrollierte die Stadt die wichtigsten landesherrlichen Rechte und war de facto autonom. Die Gesamtstadt übernahm den Großteil der Finanzverwaltung Braunschweigs. Mack (1889), S. 12 f., 18-57; Bohmbach (1969), S. 119 f. 
ger Rentenmarkt in einen Zeitraum, in dem der Rentenmarkt sehr aktiv war und auswärtiges Kapital in bemerkenswertem Maße einbezog. Wir haben uns auf Neurenten beschränkt, die über Ortsangaben einen auswärtigen Rentenkäufer identifizieren, und Konversionen, Übertragungen, Verkäufe und Umschreibungen nicht einbezogen. Zum Braunschweiger Rentenmarkt allgemein liegt mit diesem Band auch ein Datensatz zu den verkauften Renten und ihren Zinssätzen vor, der ebenfalls in die Analyse mit einbezogen und für die Jahre ergänzt wurde, die im Datensatz nicht abgedeckt sind..$^{\circ}$ Der Endpunkt 1599 ergibt sich aus den stark rückläufigen Rentenverkäufen der Stadt seit den 1570er Jahren. Nach 1587 sind bis 1599 insgesamt nur noch vier Rentenverkäufe verzeichnet, dabei keine an Auswärtige.

Im Folgenden werden wir auf die räumliche Verteilung der überlokalen Rentenverkäufe der Stadt Braunschweig eingehen, diese mit Handels- und politischen Netzwerken in Verbindung bringen und auch die Käufergruppen vorstellen. Daten zu einzelnen Rentenkäufern, die auf mehreren Märkten aktiv waren, erlauben es uns zudem, den Braunschweiger Markt in weitere Zusammenhänge einzubetten und anfänglich das Kapitalnetzwerk zu skizzieren, in das Braunschweigs Rentenmarkt eingebunden war. Anschließend wird die Entwicklung der Anteile auswärtiger Rentenkäufer über die Zeit hinweg vorgestellt. Zuletzt soll es um die Natur der Kreditbeziehungen gehen: Während die wirtschaftlichen Motivationen auswärtiger Rentenkäufer anhand von Zinssatzentwicklungen diskutiert werden, wird zuletzt auf die politischen Beweggründe von Personen wie Körperschaften während außenpolitischer Krisen eingegangen. Der letzte Abschnitt fasst unsere Ergebnisse zusammen.

\section{Die Außenbeziehungen des Braunschweiger Rentenmarktes: räumliche Verteilung und Käufergruppen}

Nach Auskunft der ,Weddeschatbücher' wurden im Zeitraum von 1361 bis 1599 in 201 von 238 Jahren frei handelbare Renten verkauft, davon in 130 Jahren Renten an Auswärtige. Insgesamt konnten in den ,Weddeschatbüchern' 4.057 Neurentenverkäufe gezählt werden; 1.208 Rentenbriefe zwischen 1390 und 1587 verzeichnen auswärtige Rentenkäufer. Nur selten kamen die Käufer einer Stadtrente aus verschiedenen Orten. Wir konnten in den Verträgen insgesamt 115 Orte identifizieren, 2,9 Prozent der Ortsangaben sind nicht zuzuordnen (37 Beobachtungen). Stellt man die Rentenverkäufe an Auswärtige allen verkauften Wiederkaufsrenten gegenüber, liegt der Durchschnitt bei 20 Prozent an auswärtigen Käufen pro Jahr.

Entfernung ist ein bestimmender Faktor für den überstädtischen Rentenkauf. Insgesamt bestätigt unsere Auswertung der räumlichen Verteilung der Braunschweiger Rentenkäufe enge Kapitalbeziehungen innerhalb der sächsisch-wendischen Städtegruppe, vor allem mit Hamburg, Lüneburg und Lübeck, weiter auch Bremen, Hannover und 
Hildesheim. ${ }^{31}$ Mit zunehmender Entfernung nimmt die Anzahl der Verträge ab (s. Abbildung 1). Setzt man eine Tagesreise oder mittlere Transportentfernung mit $30 \mathrm{~km}$ an, ${ }^{32}$ so liegen die Herkunftsorte der Geldgeber meist weniger als zwei Tagesreisen entfernt. Allerdings wurden nur zehn Prozent der Verträge innerhalb einer Tagesentfernung abgeschlossen; fast zwei Drittel der Rentenverträge wurden mit Käufern aus Städten abgeschlossen, die 30 bis $60 \mathrm{~km}$ von Braunschweig entfernt lagen. Der allergrößte Teil der Beobachtungen liegt im Dreieck Magdeburg, Göttingen und Celle. Ein Großteil der über die Stadt hinausreichenden Kreditbeziehungen war also regionaler Natur, was sich durch das Zusammenspiel von Kapitalverfügbarkeit und zunehmenden Transaktionskosten erklären lässt, die bei der Vermittlung und Administration der Renten entstanden.

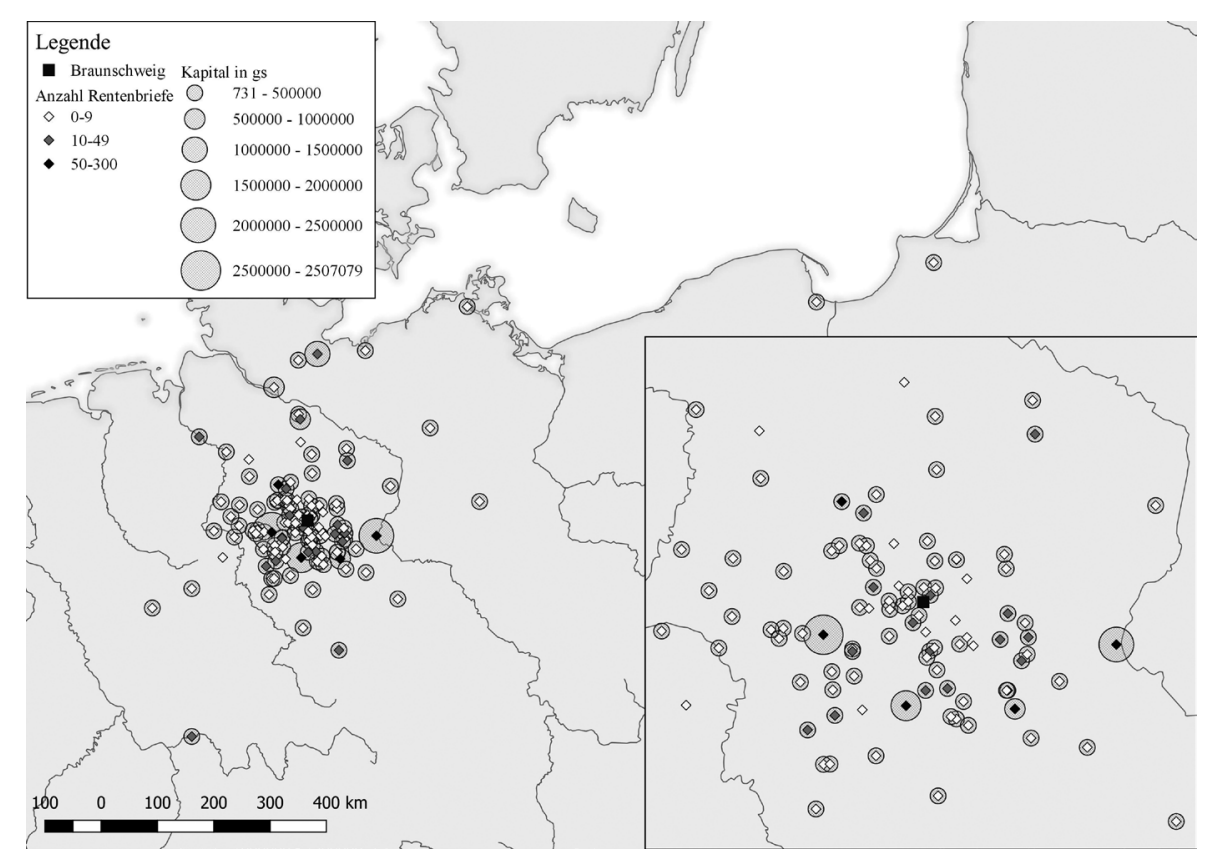

Abbildung 1: Auswärtige Rentenkäufer auf dem Braunschweiger Rentenmarkt: Herkunftsorte, Frequenz zwischenstädtischer Beziehungen und Gesamthöhe des Kapitals im Überblick Quelle: nach Tabelle 2 im Anhang. (C) Angela Huang.

Dabei konzentrieren sich die Vertragsbeziehungen auf wenige Orte: ${ }^{33}$ je größer und wirtschaftlich wichtiger der Herkunftsort des Rentenkäufers, desto mehr Verträge wurden geschlossen. Nur für 26 Orte liegen zehn oder mehr Beobachtungen vor, was einem Fünftel der nachgewiesenen Orte mit Kreditbeziehungen zur Stadt Braunschweig ent- 
spricht. Die Rentenkäufe von Käufern dieser Orte machen allerdings über 8o Prozent der Beobachtungen aus. Mehr als die Hälfte der Verträge wurde an Käufer aus nur fünf Städten ausgestellt: Knapp 24 Prozent der Beobachtungen entfallen allein auf Hildesheim, weiter je neun Prozent auf Goslar und Magdeburg, sechs Prozent auf Halberstadt und vier Prozent auf Celle. Intensivere überregionale Beziehungen bestanden zu Lübeck (drei Prozent), Bremen (1,4 Prozent) Frankfurt a. M. (1,2 Prozent) und Lüneburg (1,1 Prozent).

Auch das Kapitalvolumen konzentriert sich auf wenige Städte: Über 50 Prozent des von Auswärtigen in Braunschweig angelegten Kapitals kommt aus nur vier Städten bzw. zwei Drittel aus sieben Städten, die von den nach Anzahl der Verträge führenden Märkten teilweise verschieden sind: ${ }^{34}$ Es führt wieder Hildesheim (17 Prozent), gefolgt von Magdeburg (16 Prozent), Goslar (elf Prozent), Lübeck (sieben Prozent), Hamburg (sechs Prozent), Lüneburg (fünf Prozent) und Halberstadt (fünf Prozent). Auch wenn die Kapitalangaben unvollständig sind, illustrieren sie doch Unterschiede der zwischenstädtischen Beziehungen: Überregionale Kreditgeber kauften weniger Renten, legten aber je Rentengeschäft größere Summen an, so die Lübecker mit drei Prozent der Rentenkäufe, aber sieben Prozent der Kapitalien. Insbesondere die Höhe des geliehenen Kapitals unterstreicht die interregionalen Beziehungen der Stadt Braunschweig - hier sind Märkte wie Frankfurt, Lüneburg, Hamburg und Lübeck von ähnlicher Bedeutung wie die regionalen Zentren.

Woraus ergibt sich aber die Intensität der Kapitalbeziehungen mit bestimmten Orten? Hier zeigen sich deutliche Übereinstimmungen mit wirtschaftlichen und politischen Strukturen, in welche die Stadt Braunschweig regional und überregional eingebunden war. Die Städte, aus denen die meisten Kreditgeber kamen, sind wichtige Handelspartner Braunschweigs innerhalb der Region und des hansestädtischen Netzwerks: ${ }^{35}$ Der Raum zwischen Weser und Elbe, Harz und Lüneburger Heide war Einkaufsgebiet der Braunschweiger Kaufleute für Getreide, industrielle Rohstoffe (z. B. Wolle, Flachs), Halbfabrikate und Fertigwaren. Insbesondere mit Goslar bestanden enge Handelsverbindungen, da von hier die Harzmetalle zur Verarbeitung durch das Braunschweiger Gewerbe eingeführt wurden. Die Beziehungen zu den Märkten in der Region wie auch darüber hinaus bestanden zudem auch durch die Exportproduktion der Stadt, namentlich Metallwaren, Textilien, Leder und Bier. Der regionale Absatz war dabei vorrangig, wenn auch Metallwaren und Textilien nachweislich über Lüneburg, Lübeck und Hamburg in den überregionalen Handel eingebracht wurden. Zum Einkauf von Fernhandelswaren waren Braunschweiger Bürger in den Messestädten Antwerpen, Frankfurt und Leipzig aktiv.

Die Kreditbeziehungen spiegeln diese Handelsbeziehungen nicht eins zu eins wider, bewegen sich jedoch deutlich entlang der durch den Warenhandel gezeichneten Linien.

34 Die Angaben zu Kapital wurden soweit möglich in gs umgerechnet, um vergleichbare Werte zu schaffen, vgl. Tabelle im Anhang. Wir danken Prof. Oliver Volckart für die Zurverfügungstellung der SilbergehaltDaten zur Umrechnung des Kapitals. 
Ist es „unbestritten, dass der Rentenkauf im Reich auch als Kreditform zur Organisation des Handels gedient hat ", ${ }^{36}$ so fehlen Belege für die Beziehung zwischen Rentenmärkten und Warenmärkten. Es kann jedoch für die Stadtrenten die These aufgestellt werden, dass die Handelsnetzwerke auch die Infrastruktur für auswärtige Rentengeschäfte stellten.

Braunschweig war innerhalb der Gruppe der ,sächsischen` oder ,überheidischen` Städte auch politisch führend und pflegte enge Beziehungen mit den zeitweise bis zu 25 regionalen Bündnispartnern, worunter neben Braunschweig in absteigender Reihenfolge Hildesheim, Hannover, Göttingen, Einbeck, Goslar, Magdeburg und Halberstadt die aktivsten Städte waren. Darüber hinaus war Braunschweig auch Hansestadt und daher in ein weiterreichendes Städtenetzwerk und Märktesystem eingebunden. Braunschweig war in der Hanse seit der Zeit um 1500 Vorort bzw. Quartiersstadt und vermittelte zwischen den Städten der Region und der Hanse. Die Stadt nahm an Tagfahrten und hansischen Schutzbündnissen teil. Gerade die gemeinsame Verteidigung gegen die Landesherren soll zentral gewesen sein für Braunschweigs verstärktes Interesse an der hansischen Gemeinschaft im 15. und 16. Jahrhundert. ${ }^{37}$ Braunschweigs hansische Aktivität erklärt nicht die Intensität, aber die Reichweite der Braunschweiger Stadtrentengeschäfte entlang der Ostseeküste nach Stralsund, Wismar, Danzig und Königsberg. Wie zu zeigen sein wird, waren diese politischen Verbindungen der Städte von mindestens ebenso großer Bedeutung für die Beteiligung Auswärtiger am Braunschweiger Rentenmarkt wie die Wirtschaftsbeziehungen der Stadt und einzelner Akteure.

Bislang wurden die auswärtigen Rentenkäufer als eine geschlossene Gruppe dargestellt. Um die über die Stadt hinausreichenden Kapitalverflechtungen weiter ausdifferenzieren zu können, unterscheiden wir im Weiteren die Käufer in Körperschaften und Privatpersonen einerseits und geistliche oder weltliche Akteure andererseits. Grundsätzlich lässt sich dabei feststellen, dass die von der Forschung identifizierten führenden Gruppen im innerstädtischen Rentenkauf auch den überlokalen Rentenkauf dominierten: Rentenkäufer waren vor allem Bürger aus der wirtschaftlich und politisch führenden Schicht der Städte, weiter Geistliche und kirchliche Körperschaften. Zu ergänzen sind andere Ratskollegien. ${ }^{38}$

36 Gilomen (1984), Zitat S. 58.

37 Zu Braunschweigs Bündnistätigkeit mit den sächsischen Städten und der Hanse im 14. und 15. Jh. Puhle (1985), eine Liste der sächsischen Städte nach ihrer Bündnisaktivität auf S. 208; Steinführer (2017), S. 2024, 32; Dollinger (1998), S. 130, 159-160. Braunschweig nahm an mehr als einem Drittel der 172 bekannten Hansetage seit 1358 teil und gehörte zu den Städten, die bis zum letzten Hansetag 1669 an den Versammlungen teilnahmen, HR I 1, Nr. 212; HR I 2, Nrn. 263, 266; HR I 3, Nr. 443; HR I 4, Nrn. 38, 441; HR I 5 , Nr. 705; HR I 6, Nrn. 68, 397, 556; HRI 7, Nrn. 156, 712; HR II 1, Nr. 321; HR II 2, Nr. 439; HR II 3, Nrn. 288, 627, 649; HR II 4, Nrn. 161, 248, 458; HR II 6, Nr. 356; HR II 7, Nr. 389; HR III 3, Nr. 353; HR III 4, Nr. 79; HR III 6, Nr. 188; HR III 7, Nrn. 39, 108; HR IV 2, Nr. 86; Höhlbaum (1896); AHL ASA Externa Hanseatica, Nrn. 203 f., 207, 209, 211, 213, 218, 224a, 230, 235-237, 247; Stadtarchiv Braunschweig, B III 4:22, 4:34, 4:45.

38 Vgl. Albers (1939), S. 43-47; Eberhardt (1996), S. 46-50, Tabelle S. 52; Fuhrmann (2016), S. 47-168; Beyer (1901), S. 119; Bohmbach 1969, 126-129; Lorenzen-Schmidt (1979-1980), S. $111 \mathrm{f}$. 
Von Braunschweigs auswärtigen Rentenkäufern konnten wir gut 40 Prozent als Privatpersonen identifizieren - diese waren nach Anzahl der Verträge die wichtigste Gruppe. Es folgen mit gut 30 Prozent der Verträge geistliche Körperschaften und mit weiteren gut 20 Prozent geistliche Einzelpersonen. Anlagen städtischer Körperschaften und hier vor allem von Räten anderer Städte erscheinen mit nur knapp fünf Prozent der Rentenkäufe vergleichsweise unbedeutend - allerdings stellen sie gut 30 Prozent des angelegten Kapitals, während sich hier die Bedeutung von Privatpersonen und geistlichen Körperschaften relativiert (s. Tabelle 1).

Tabelle 1: Käufergruppen nach Anzahl der Beobachtungen und in Anteilen am Kapital in Silber

\begin{tabular}{lrrrrrr}
\hline Käufergruppe & $\begin{array}{r}\text { o Entfer- } \\
\text { nung }(\mathbf{k m})\end{array}$ & $\begin{array}{r}\text { Anzahl } \\
\text { Verträge }\end{array}$ & $\begin{array}{r}\text { Verträge } \\
(\%)\end{array}$ & $\begin{array}{r}\text { Beobachtun- } \\
\text { gen Kapital }\end{array}$ & $\begin{array}{r}\text { Summe Ka- } \\
\text { pital (gs) }\end{array}$ & $\begin{array}{r}\text { Summe } \\
\text { Kapital } \\
(\%)\end{array}$ \\
\hline $\begin{array}{l}\text { Adel } \\
\text { Körperschaft, } \\
\text { geistlich }\end{array}$ & 51,9 & 403 & 32,0 & 2 & 63.422 & 0,4 \\
$\begin{array}{l}\text { Körperschaft, } \\
\text { städtisch }\end{array}$ & 126,7 & 57 & 4,0 & 227 & 2.065 .773 & 14,2 \\
$\begin{array}{l}\text { Geistlicher } \\
\text { Stand }\end{array}$ & 52,0 & 280 & 22,0 & 349 & 4.473 .133 & 30,8 \\
$\begin{array}{l}\text { Privatperson } \\
\text { Gesamt- }\end{array}$ & 61,1 & 534 & 42,0 & 49 & 3.403 .445 & 23,4 \\
ergebnis & 59,1 & 1.279 & 100,0 & 347 & 4.534 .142 & 31,2 \\
\hline
\end{tabular}

Quelle: StA Braunschweig, B I 12, Bde. 1-6.

Einige in Braunschweig belegte auswärtige Käufer konnten wir auch auf den Rentenmärkten von Bremen, Hamburg, Hannover, Hildesheim und Lüneburg nachweisen Märkte, die beispielhaft Braunschweigs regionale und überregionale Kapitalbeziehungen repräsentieren. Insgesamt konnten wir 14 weltliche und zehn geistliche Personen sowie sechs weltliche und 22 geistliche Institutionen aus 21 Orten identifizieren, die in Braunschweig und wenigstens einer anderen Stadt Stadtrenten erwarben (s. Abbildung 2).

Diese Daten geben uns einen ersten Einblick in die Kapitalanlagen einzelner Kapitalgeber über einen Rentenmarkt hinaus und zeigen bereits Gemeinsamkeiten in der Herkunft des Kapitals verschiedener städtischer Rentenmärkte. Einige Orte und Käufergruppen zeichnen sich durch stärkere Aktivität auf verschiedenen Märkten aus. Käufer aus Celle, Hildesheim und Lübeck führen mit Rentenkäufen auf vier der fünf hier einbezogenen Rentenmärkte. Im Durchschnitt erwarben die auf mehreren Märkten dokumentierten Käufer insgesamt um die sechs Renten.

Auf weltlicher Seite ist allein der Lübecker Rat auf mehr als zwei Rentenmärkten als Käufer greifbar. Die Hildesheimer Kirchen stechen als Käufer hervor: Die Domkirche, St. Andreas, St. Crucis und St. Maurici kauften jeweils Renten auf drei Märkten. Das Karthäuserkloster St. Marien zu Hildesheim war sogar auf vier der fünf Rentenmärkte 
als Käufer aktiv. Auch unter den geistlichen Personen ist Hildesheim besonders präsent. Der Kanoniker Johann Lerte taucht dabei auf drei Märkten auf und ist überhaupt die einzige Person, die auf mehr als zwei Märkten aktiv war. Darüber hinaus waren das Kloster Wienhausen mit 20 und das Kloster Wöltingerode mit 21 Transaktionen auf je drei Märkten vertreten, v. a. Braunschweig, dann auch Hannover und Lüneburg sowie Hildesheim und Lüneburg.

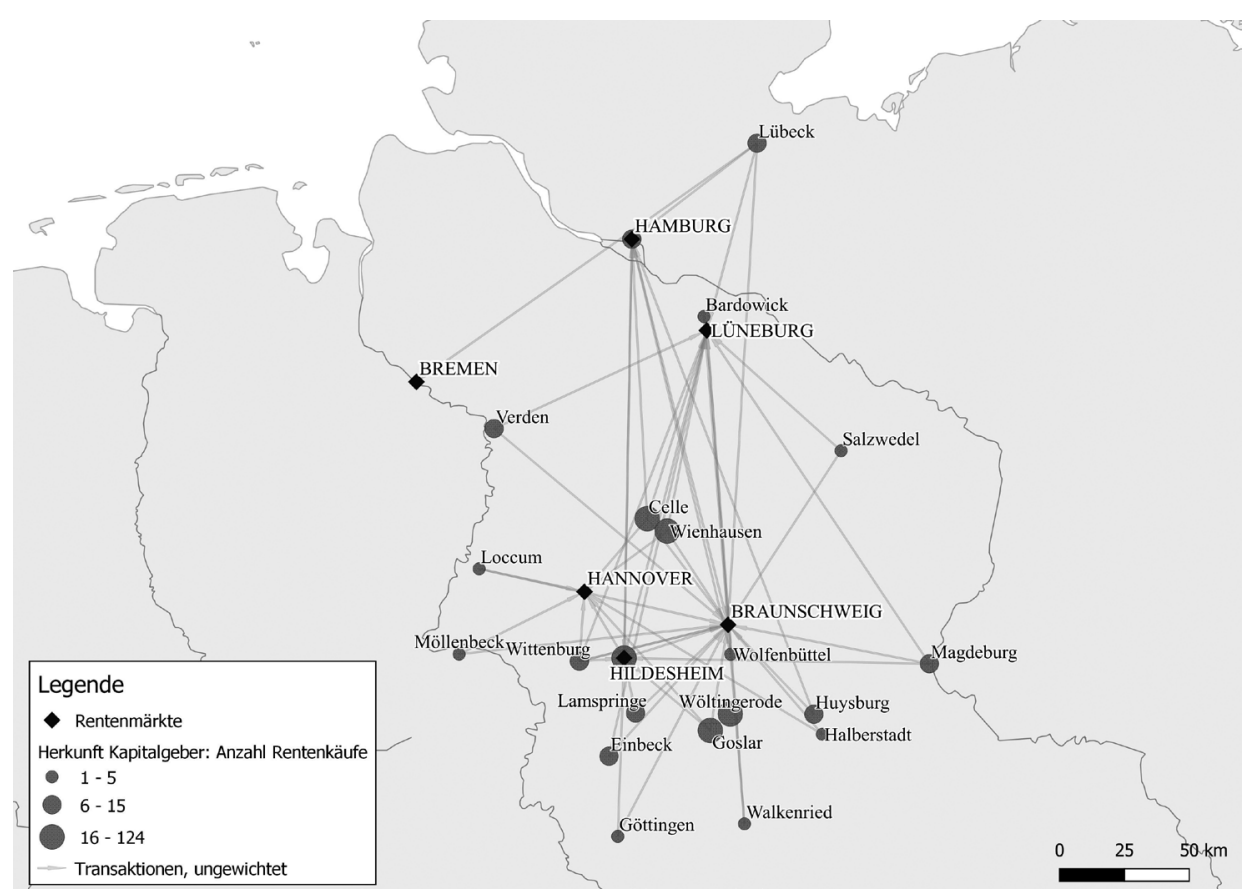

Abbildung 2: Anlagen von auswärtigen Rentenkäufern auf mehreren Märkten nach Auswertung von Braunschweig, Bremen, Hamburg, Hannover, Hildesheim und Lüneburg, 15.-16. Jh.

Quelle: nach Tabelle 4 im Anhang. (C) Angela Huang.

Was den Aktionsradius der Rentenkäufer anbelangt, so betrug die Entfernung zwischen dem Herkunftsort des Käufers und den kreditnehmenden Städten durchschnittlich $70 \mathrm{~km}$ (s. Tabelle 2). Sie war am höchsten bei den Käufen von Stadträten, gefolgt von weltlichen Personen. Bei weltlichen Personen dürfen wir vermuten, dass es sich hier höchstwahrscheinlich um die Oberschicht der Städte handelte, die durch ihre kaufmännsische Tätigkeit entsprechende Kapitalüberschüsse und wirtschaftliche Interessen auf den jeweiligen Märkten hatte. Es folgen Geistliche und geistliche Körperschaften. 
Tabelle 2: Auswärtige Rentenkäufer, die auf mindestens einem weiteren Markt nachgewiesen werden konnten

\begin{tabular}{cccc}
\hline & $\begin{array}{r}\text { durchschn. Entfernung } \\
(\mathbf{k m})\end{array}$ & $\begin{array}{r}\text { durchschn. Anzahl } \\
\text { an Rentenbriefen }\end{array}$ & $\begin{array}{r}\text { durchschn. Aktivi- } \\
\text { tät in Jahren }\end{array}$ \\
\hline Privatpersonen & 71 & 4,5 & 13,6 \\
Weltlich & 81 & 4,0 & 9,4 \\
Geistlich & 63 & 5,0 & 14,5 \\
Geistliche Körperschaft & 67 & 7,0 & 67,0 \\
Räte & 105 & 8,5 & 46,5 \\
\hline
\end{tabular}

Quelle: StA Braunschweig, B I 12, Bde.1-6.

Selbst in diesem relativ kleinen Testdatensatz von nur fünf Märkten können wir also eine nennenswerte Anzahl an Käufern ausmachen, die in mehr als einer Stadt Stadtrenten erwarben und ihr Kapital räumlich streuten. Die von uns zusammengestellten Daten zu Käufern auf mehreren Rentenmärkten heben die regelmäßige Einbindung nicht nur von städtischen Akteuren, sondern auch von außerhalb der Städte gelegenen Klöstern in städtische Kreditnetzwerke hervor. Die hier nachvollzogenen Kapitalbeziehungen reichen - im Unterschied zu der räumlichen Verteilung allein der auswärtigen Käufer auf dem Braunschweiger Rentenmarkt - über die Region der jeweiligen Märkte deutlich hinaus. Aktivitäten einzelner offenkundig kapitalstarker Käufer auf mehreren Rentenmärkten gehen einher mit einer größeren Distanz ihrer Kapitalbeziehungen.

\section{Die Entwicklung der Beteiligung Auswärtiger über die Zeit}

Wie die eingangs vorgestellten Forschungen zu wechselnden Anteilen Auswärtiger an städtischen Rentenverkäufen gezeigt haben, ist bei der Einschätzung der Rolle Auswärtiger die Entwicklung über die Zeit hinweg zu beachten. Denn Renten dienten oft der Finanzierung außerordentlicher Projekte und Städte sollen Rentenverkäufe an Auswärtige im Normalfall vermieden haben. Wir wollen uns im Folgenden der zeitlichen Entwicklung der Braunschweiger Stadtrentenverkäufe zuwenden, um einzuschätzen, ob dieses Urteil auch für den Braunschweiger Rentenmarkt gilt.

In Braunschweig wurden im 15. und 16. Jahrhundert fast in jedem Jahr Renten verkauft, aber die Zahl der verkauften Renten schwankte stark. Im hier untersuchten Zeitraum von 1361 bis 1599 bewegten sich die Rentenverkäufe insgesamt auf einem niedrigen Niveau und nahmen nur zeitweise in bemerkenswertem Umfang zu. Der Durchschnitt lag bei 17 Stadtrentenverkäufen pro Jahr, für Auswärtige bei fünf verkauften Renten; der Modalwert liegt bei jeweils nur einer Rente pro Jahr. Ein Spitzenwert von 228 Stadtrentenverkäufen, davon 141 Rentenverkäufen an Auswärtige (nahezu 62 Prozent) ist für das Jahr 1493 zu beobachten. Was die großen Trends über das 15. und 16. Jahrhundert hinweg anbelangt, so zeigt die Grafik (s. Abbildung 3), dass regelmäßige Rentenverkäufe erst mit den 1380ern einsetzen und bis Ende des 16. Jahrhunderts fast durchgehend erfolgen, 
bis auf eine Lücke in den 1470ern. Mit steigenden Rentenverkäufen steigt auch der Anteil auswärtiger Käufer, wobei ein auffälliger Unterschied zwischen dem 15. und 16. Jahrhundert besteht: Der Anteil der Auswärtigen ist bis ins späte 15. Jahrhundert deutlich geringer. Mit den 1480ern, 100 Jahre nach Einsetzen regelmäßiger Rentenverkäufe, setzt ein deutlicher Wandel in der Beteiligung auswärtiger Käufer ein (s. Tabelle 3). Bei weiterhin schwankender Anzahl an Rentenverkäufen steigt der Anteil der Auswärtigen und verbleibt auf einem höheren Niveau von gut 30 Prozent. Nur in den Jahren 1540 bis 1546 gelang es der Stadt, weniger als zehn Renten zu verkaufen und mehrere Jahre in Folge kein auswärtiges Kapital aufzunehmen.

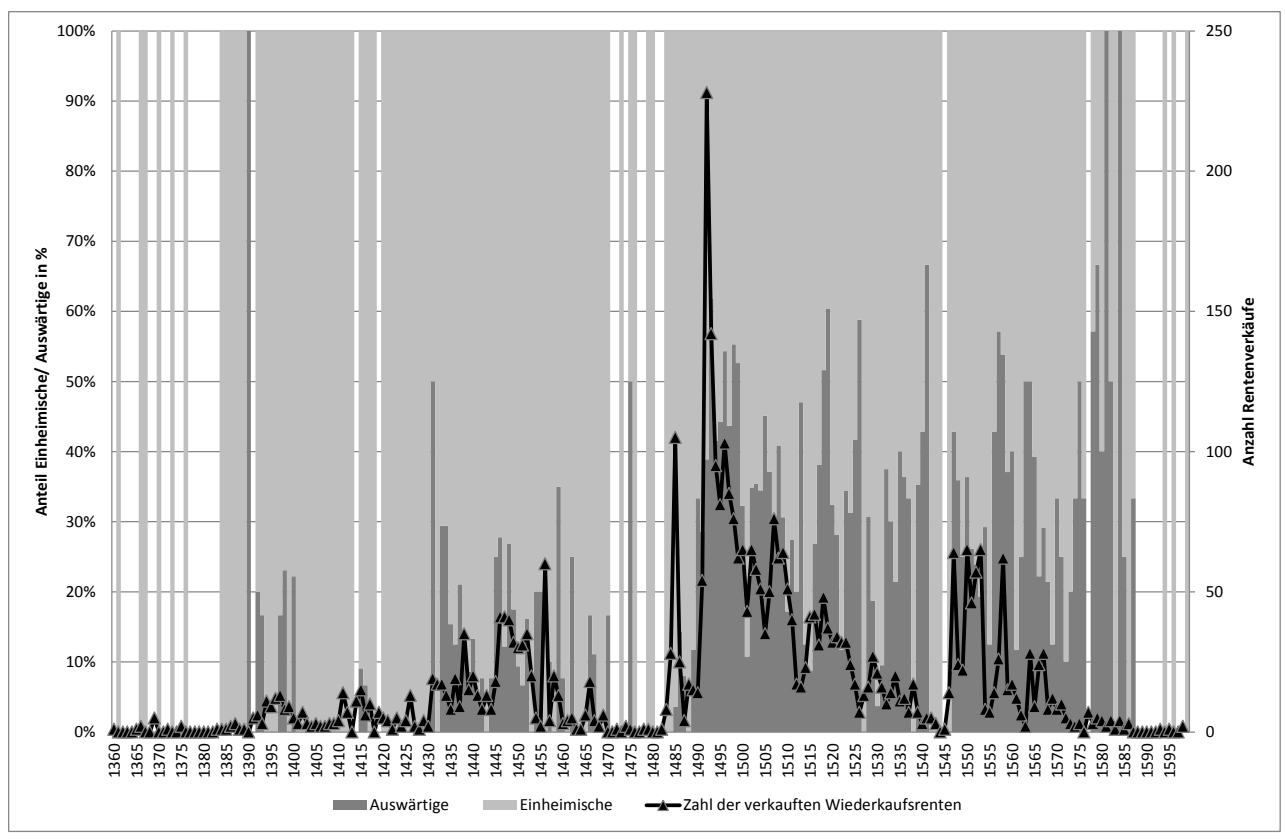

Abbildung 3: An Auswärtige verkaufte Wiederkaufsrenten der Stadt Braunschweig pro Jahr und Anteil der auswärtigen Rentenkäufer an den verkauften Wiederkaufsrenten in Prozent Quelle: nach Tabelle 1 im Anhang.

Tabelle 3: Verkäufe von wiederkäuflichen Renten durch die Stadt Braunschweig, 1384-1484 und 1485-1599

\begin{tabular}{lrr}
\hline & $1384-1484$ & $1485-1599$ \\
\hline Summe verkaufter Verträge & 919 & 3.138 \\
Durchschnittl. Anzahl verkaufter Renten & 9 & 30 \\
Maximum verkaufter Renten & 60 & 228 \\
Anteil Auswärtiger in \% & 8 & 31 \\
\hline
\end{tabular}

Quelle: Tabelle 1 im Anhang. 
Über die hier betrachteten 240 Jahre nahmen die Stadtrentenverkäufe wie auch die Anteile Auswärtiger dabei in drei Zeiträumen zunächst sprunghaft zu, so ca. 1430 bis 146o, 1484 bis ca. 1540 und zuletzt, nur wenige Jahre später, 1547 bis 1572 . Vor allem das Ende dieser Phasen ist dabei kaum fest zu benennen, da die Rentenverkäufe nach mehr oder minder kurzen Anstiegen allmächlich wieder auf ein niedriges Niveau zurückkehren.

Die Höhepunkte der Rentenverkäufe fallen zusammen mit Zeiten innerer und äußerer Konflikte (s. Tabelle 4). Hervorzuheben ist als Kostenverursacher neben Bündnisverpflichtungen gegenüber in Gefahr geratenen Nachbarstädten vor allem das Ringen um städtische Unabhängigkeit seit 1491: Nach dem Rücktritt Herzog Wilhelms des Jüngeren 1491 ist die Folgezeit bis zur Unterwerfung der Stadt 1671 gezeichnet durch Konflikte mit den Herzögen, angefangen mit der ,Große Stadtfehde' gegen Herzog Heinrich den Jüngeren 1492-1494. Im Nachgang dieser inneren und äußeren Belastungen erleben die Rentenverkäufe der Stadt zwischen circa 1490 und circa 1520 ihren absoluten Höhepunkt. Die extrem hohen Rentenverkäufe während der ,Großen Stadtfehde ' und in der Folgezeit zeigen eindrucksvoll, in welchem Ausmaß vor allem die fast durchgehenden Auseinandersetzungen mit den Herzögen bis zum Ende der städtischen Unabhängigkeit 1671 den städtischen Haushalt belasteten. Der Glaubenswechsel verstärkte den Konflikt mit dem Herzog, bis es 1531 endgültig zum Bruch kam und sich Braunschweig dem Schmalkaldischen Bund anschloss. Kosten entstanden in der Folgezeit durch Braunschweigs Beitrag zum Bundesheer und den Ausbau der städtischen Befestigungsanlagen, schließlich Entschädigungsgelder, die 1553 im Rahmen des Friedens mit dem Herzog gezahlt werden mussten.

Tabelle 4: Innere und äußere Konflikte der Stadt Braunschweig, Mitte des 15. Jahrhunderts bis in die zweite Hälfte des 16. Jahrhunderts

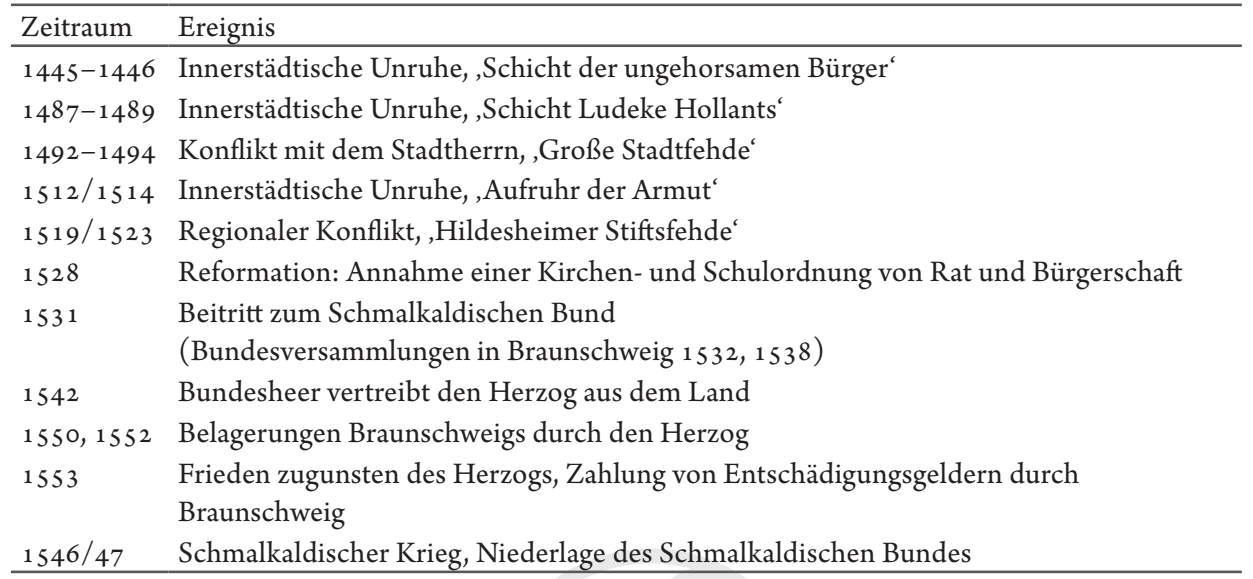
Quellen: nach Moderhack (1985), S. 31-38, 40-44; Dürre (1861), S. 219-255; Spiess (1966), S. 19-95.

Auch in Braunschweig führten also äußere Konflikte zu außerordentlichen Kosten, die durch Rentenverkäufe, in zunehmendem Maße auch an Auswärtige, getragen werden mussten. Seit dem späteren 15. Jahrhundert brachten diese Kapitalaufnahmen Braun- 
schweigs Haushalt in eine andauernde Schräglage, die auch zahlreiche innere Unruhen mit verursachte. Besonders die Ausgaben der oberdeutschen Städte für den Schmalkaldischen Bund in den 1540er Jahren wurden als „den Finanzrahmen aller bis dahin durchgeführten kommunalen Kriegsunternehmungen sprengende Aufwendungen“ bezeichnet, wobei die zeitliche Verteilung der Braunschweiger Rentenverkäufe für eine größere Belastung durch die Konflikte mit dem Stadtherrn spricht. ${ }^{39}$

Geistliche Körperschaften wurden bereits als bedeutende Kapitalgeber Braunschweigs und anderer Städte herausgestellt. So wurde etwa für den Kieler Rentenmarkt festgestellt, dass mit der Reformation die geistliche Anlagetätigkeit vollständig abbrach..$^{40}$ Deswegen muss bei einer Beschäftigung mit Braunschweigs auswärtigen Rentenkäufern im 16. Jahrhundert auch nach den Folgen der Reformation für das städtische Kreditwesen gefragt werden. Weniger als ein Viertel der auswärtigen Renten wurden dabei in nachreformatorischer Zeit verkauft, wobei sich dieser geringe Anteil auch aus den hohen Rentenverkäufen der 1490er Jahre ergibt. Nichtsdestoweniger können wir feststellen, dass nicht nur die absolute, sondern auch die relative Beteiligung von geistlichen Körperschaften und Personen nach der Reformation stark zurückging (s. Tabelle 5).

Tabelle 5: Anteile der Rentenkäufergruppen vor und nach der Reformation

\begin{tabular}{lrrrrrr}
\hline \multirow{2}{*}{ Rentenkäufer } & \multicolumn{2}{c}{ Vorreformatorisch } & \multicolumn{2}{c}{ Nachreformatorisch } & \multicolumn{2}{c}{ Gesamtergebnis } \\
& Verträge & in $\%$ & Verträge & in \% & Verträge & in \% \\
\hline Adel & 4 & 0,4 & 1 & 0,4 & 5 & 0,4 \\
Geistlicher Stand & 257 & 25,8 & 23 & 8,1 & 280 & 21,9 \\
Körperschaft, geistlich & 370 & 37,1 & 33 & 11,7 & 403 & 31,5 \\
Körperschaft, städtisch & 51 & 5,1 & 6 & 2,1 & 57 & 4,5 \\
Privatperson & 314 & 31,5 & 220 & 77,7 & 534 & 41,8 \\
\hline Gesamtergebnis & 996 & 100,0 & 283 & 100,0 & 1.279 & 100,0 \\
\hline
\end{tabular}

Quelle: StA Braunschweig, B I 12, Bde. 1-6.

Die bereits als Kapitalgeber auf verschiedenen Rentenmärkten belegten Klöster illustrieren die Folgen der Reformation: Für das Kloster der Zisterzienserinnen zu Wienhausen können wir zwischen 1438 und 1507 und dann noch einmal 1555 Rentenverträge mit Braunschweig (17), Hannover (zwei) und Lüneburg (eine) nachweisen. ${ }^{41}$ Hier wurde 1527 die Reformation durchgesetzt. Die Vereinnahmung der Klostergüter durch den Herzog schwächte die wirtschaftliche Situation des Konvents nachhaltig und entsprechend ist er nicht länger als Rentenkäufer nachweisbar. Das Kloster der Zisterzienserinnen zu Wöltingerode kann mit 21 Transaktionen zwischen 1437 und 1550 ebenfalls als wichtiger Kapitalgeber für Braunschweig, Hildesheim und Lüneburg gelten. Noch 1542

39 Fouquet (1989), S. 43.

40 Lorenzen-Schmidt (1979-1980), S. 112.

41 Vgl. Tabelle 4 im Anhang. 
weigerten sich die nur noch ${ }_{25}$ Chorfrauen, die Reformation einzuführen, 1572 musste der Konvent aber schließlich gegenüber dem Herzog nachgeben. Dass hier die Reformation erst später durchgesetzt wurde erklärt, dass das Kloster bis 1550 weiterhin als Rentenkäufer überliefert ist. ${ }^{42}$ Diese Beispiele zeigen, wie Braunschweig mit der Reformation zuvor verlässliche Geldgeber wegbrachen. Der Kapitalbedarf musste nun über weltliche Käufer gedeckt werden.

\section{Zinssatzdifferenzen}

In Braunschweig war der öffentliche Haushalt, wie wir gesehen haben, seit dem späten 15. Jahrhundert nicht unwesentlich auf auswärtiges Kapital angewiesen. Es gelang der Stadt, auswärtige Kapitalien in großem Umfang zu mobilisieren, und es fragt sich, was die Rentenkäufer zur Anlage ihrer Kapitalien auf fremden Rentenmärkten bewog. Boten etwa Zinssätze wirtschaftliche Anreize für Kapitalanlagen in Braunschweig?

Höhere Zinssätze für Auswärtige sind verschiedentlich belegt. In Hamburg unterschieden sich die Zinssätze für einheimische und auswärtige Rentenkäufer im späten 15. Jahrhundert bei Wiederkaufsrenten. Einheimische erhielten höhere Zinssätze als Auswärtige (6 2/3 Prozent ggü. 4-5 Prozent) Bei Leibrentenverkäufen erhielten Auswärtige (Lübecker) allerdings mit 7 bis 8 Prozent einen höheren Zinssatz als die 6 2/3 Prozent für Einheimische, auch 2 2/3 bis 3 Prozentpunkte höher als in Lübeck zu dieser Zeit. ${ }^{43}$ Ansonsten hat die Forschung nur höhere Zinssätze für Auswärtige beobachtet. Für Köln hat Knipping 1474 im Zusammenhang mit dem Neusser Krieg Zinssatzerhöhungen von vier auf fünf Prozent (Erbrenten) bzw. von 8 1/3 Prozent auf zehn Prozent (Leibrenten) festgestellt, wobei 1478 die Zinssätze wieder auf das vorherige Niveau zurückkehren sollten. ${ }^{44}$ In Basel wurden 1448/49 Einheimischen Zinssätze von fünf oder vier Prozent angeboten, während man nach Straßburg Renten zu 6 und $62 / 3$ Prozent verkaufte. ${ }^{45}$ Vor allem in Krisenzeiten, in denen auswärtiges Kapital dringend benötigt wurde, versprechen Städte Anlegern also höhere Zinssätze.

Betrachten wir nun den Zeitraum 1485 bis 1585 näher, in dem Auswärtige einen wichtigen Anteil am Braunschweiger Rentenmarkt hatten. Erhöhte Rentenverkäufe und Anteile Auswärtiger fallen in Braunschweig zunächst generell mit erhöhten Zinssätzen für Braunschweiger Stadtrenten zusammen. Dabei sind im Normalfall nur geringfügige Unterschiede in den Zinssätzen von Einheimischen und Auswärtigen zu beobachten; zeitweise aber finden wir Zinssatzdifferenzen von 0,5 bis 1 Prozentpunkten (s. Abbildungen 4 und 5). Es bestanden somit nicht grundsätzlich bessere Konditionen für auswärtige Rentenkäufer. In Ausnahmesituationen akuten Geldbedarfs wie insbesondere im frühen 16. Jahrhundert wurden aber Auswärtigen durchaus deutlich höhere Zinssätze angebo-

42 Zur Geschichte der Klöster: Institut für Landesgeschichte.

43 Fuhrmann (2003), S. 10.

44 Knipping (1894), S. 366-368.

45 Gilomen (2003), S. $182 \mathrm{f}$. 
ten: Vergleichen wir die jährlichen Durchschnittswerte der Zinssätze im Zeitraum 1505 bis 1511, so liegen die Zinssätze auswärtiger Rentenkäufer mit fünf Prozent einen Prozentpunkt über den Zinssätzen für Einheimische. Andersherum wurden Auswärtigen in Zeiten, in denen auswärtiges Kapital nicht unbedingt benötigt wurde, auch niedrigere Zinssätze angeboten als Einheimischen, so etwa in den 1540ern, als die Rentenverkäufe zeitweise auf ein niedriges Niveau zurückkehrten, und im späten 16. Jahrhundert, als die Rentenverkäufe der Stadt kontinuierlich zurückgingen und schließlich aussetzten.

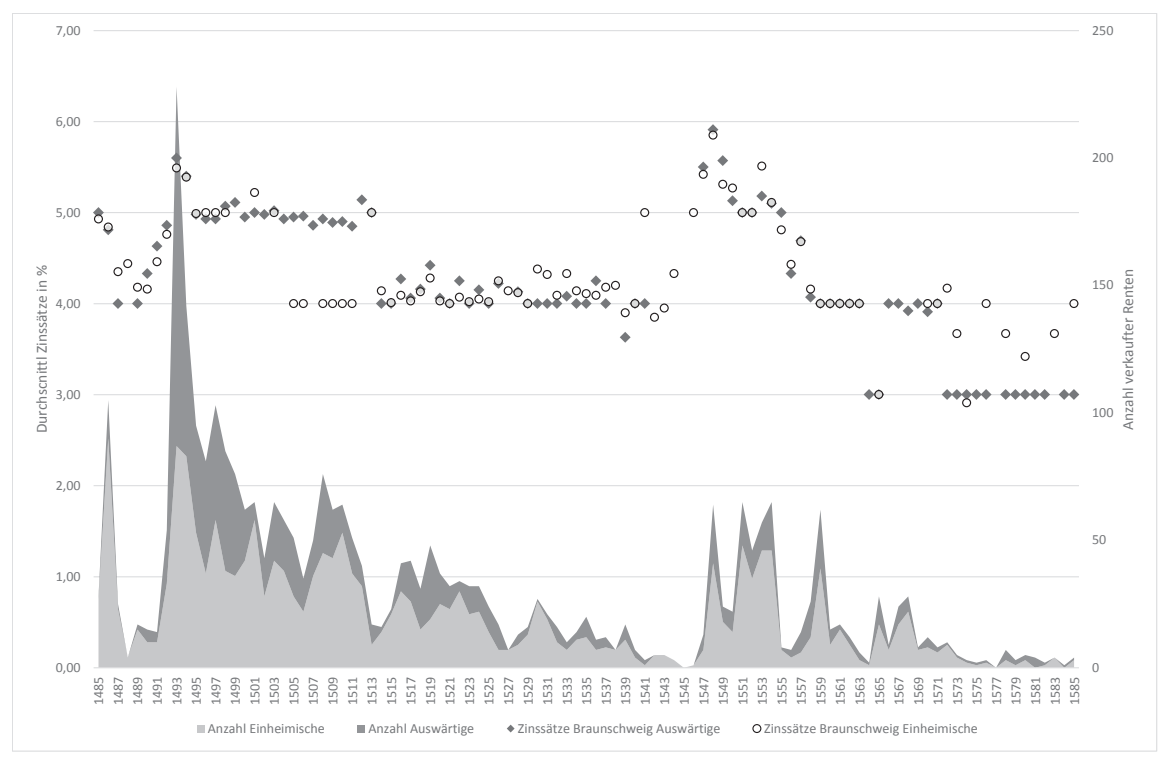

Abbildung 4: Zahl der Rentenverkäufe und Mittel der Zinssätze, 1485-1585

Quellen: nach Tabelle 1 im Anhang, StA Braunschweig B I 12, Bde. 1-6, Huang/Chilosi/Sapoznik (2019) in diesem Heft.

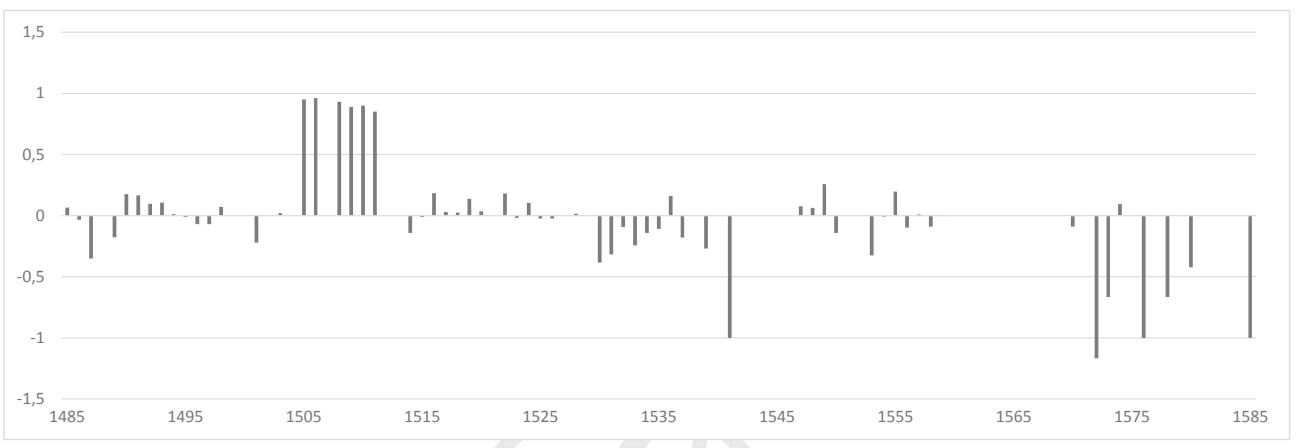

Abbildung 5: Zinssatzdifferenzen zwischen einheimischen und auswärtigen Rentenkäufern auf dem Braunschweiger Rentenmarkt, 1485-1585

Quellen: nach Tabelle 1 im Anhang, StA Braunschweig B I 12, Bde. 1-6, Huang/Chilosi/Sapoznik (2019) in diesem Heft. 
Bestanden aber für Auswärtige Anreize durch höhere Zinssätze in Braunschweig als anderswo? Vergleicht man die Braunschweiger Zinssätze mit denen anderer Städte, bestehen durchaus Abweichungen zwischen den Rentenmärkten. Die Braunschweiger Zinssätze sind dabei im hier beobachteten Zeitraum gleich wie auf den Vergleichsmärkten Bremen, Lüneburg und Hildesheim oder niedriger. Für diese Städte sind gute Zinssatzreihen verfügbar, sie gehören weiterhin zu den führenden Herkunftsorten auswärtigen Kapitals und repräsentieren verschiedene Reichweiten des Braunschweiger Rentenmarktes. Im Durchschnitt liegen der Bremer Zinssatz dabei o,75 (max. o,8) und der Lüneburger 0,3 Prozentpunkte (max. 1,7) über dem von Braunschweig. Der Hildesheimer Zinssatz liegt geringfügige o,02 Prozentpunkte unter dem von Braunschweig, allerdings mit einer maximalen Differenz von 1,4 Prozentpunkten. ${ }^{46}$

Die Braunschweiger Zinssätze sind vor allem um 1500 und im Zeitraum 1546 bis 1557 im Vergleich zu den Städten Bremen, Lüneburg und Hildesheim erhöht, also in eben den Zeiten, in denen die Rentenverkäufe und Zinssätze anstiegen - in besonderem Maße für Auswärtige. Hier sind Zinssatzdifferenzen von o,5 bis sogar über 1,5 Prozentpunkten zu verzeichnen (s. Abbildung 6). Vor allem in der zweiten Hälfte des 16. Jahrhunderts lagen die Zinssätze bei stark rückläufigen Rentenverkäufen der Stadt dann deutlich unter denen der anderen Städte, oft 1 bis 2 Prozentpunkte. Diese Unterschiede in den Zinssätzen sind durchaus bemerkenswert und könnten Anreize für auswärtige Kapitalgeber geboten haben.

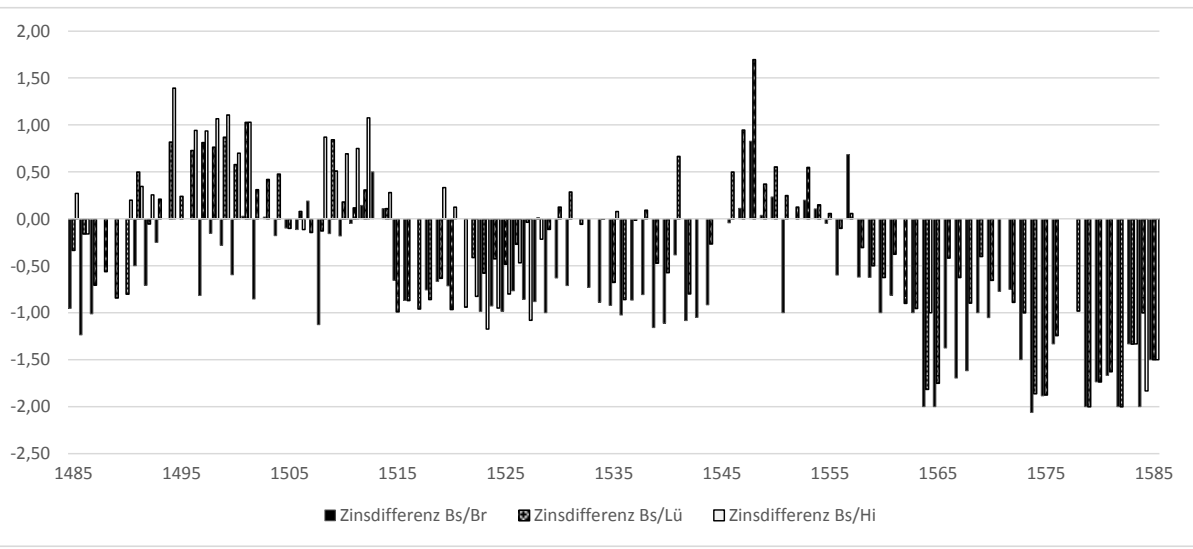

Abbildung 6: Zinssatzdifferenzen zwischen dem Braunschweiger Rentenmarkt und den Märkten Bremen, Lüneburg und Hildesheim. 1485-1585

Quellen: nach Tabelle 1 im Anhang, StA Braunschweig B I 12, Bde. 1-6, Huang/Chilosi/Sapoznik (2019) in diesem Heft.

46 Die Hildesheimer Datenreihe weist dabei allerdings große Lücken seit dem zweiten Drittel des 16. Jh. auf. Zur Datengrundlage vgl. Huang/Chilosi/Sapoznik (2019). 


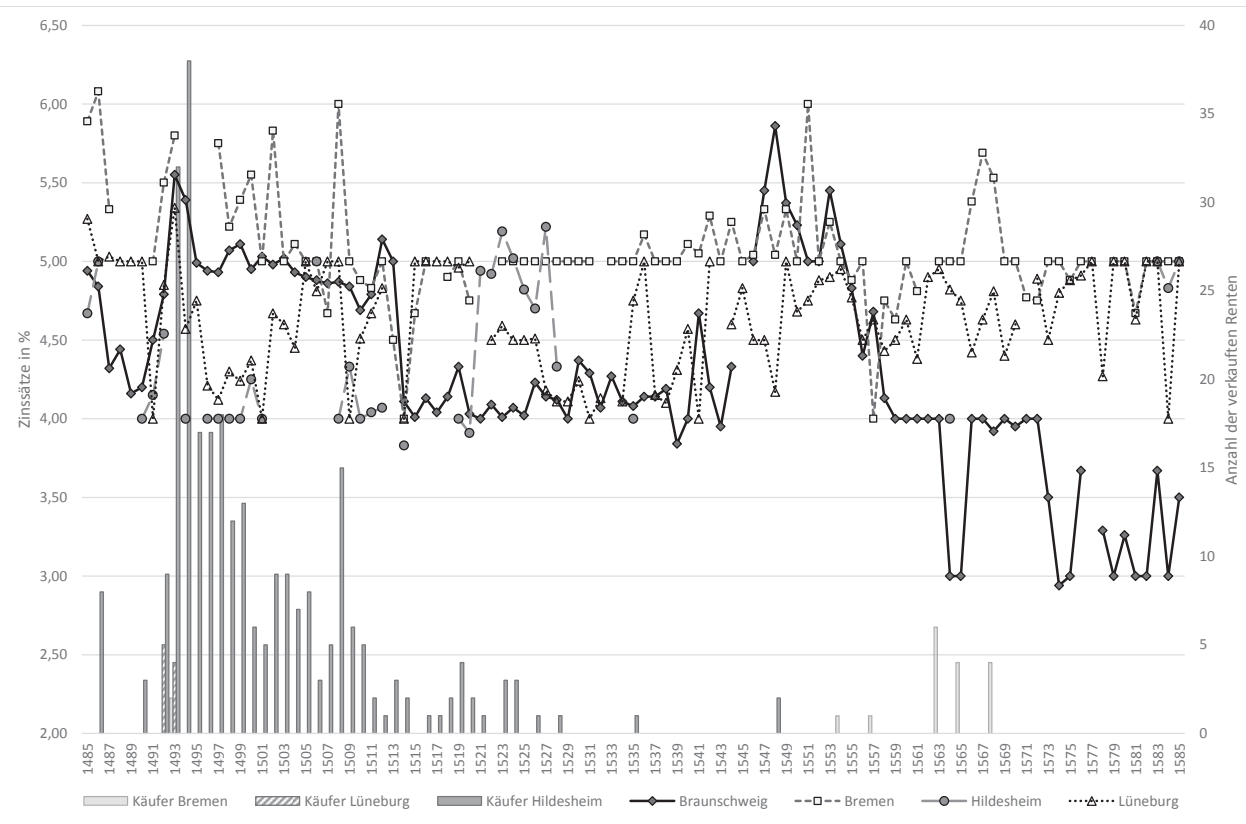

Abbildung 7: Vergleich der Zinssätze Braunschweigs mit denen der Städte Bremen, Lüneburg und Hildesheim sowie Aktivitäten von Rentenkäufern dieser Städte auf dem Braunschweiger Rentenmark, $1485^{-15} 85$

Quellen: nach Tabelle 1 im Anhang, StA Braunschweig B I 12, Bde. 1-6, Huang/Chilosi/Sapoznik (2019) in diesem Heft.

Vergleicht man nun die Zinssatzdifferenzen mit den Aktivitäten von Käufern aus den Städten Hildesheim, Lüneburg und Bremen, dann finden wir vor allem Hildesheimer Käufer in größerer Zahl auf dem Braunschweiger Rentenmarkt (s. Abbildung 7). Ihre Rentenkäufe Ende des 15. und Anfang des 16. Jahrhunderts fallen deutlich zusammen mit den im Vergleich zu ihrem Heimatort höheren Braunschweiger Zinssätzen. Im Jahre 1493 sind allerdings jeweils zwei Rentenkäufe der Stadträte von Lüneburg und Bremen belegt, die nicht durch höhere Zinssätze motiviert sein können, da sich die Zinssäzte der drei Städte in diesem Jahr fast gleichen. Insofern haben allenfalls die Hildesheimer Käufer auf höhere Zinssätze reagiert.

Etwaige Gewinne reduzierten sich allerdings durch die Kosten, die mit der Abwicklung des Finanzgeschäfts verbunden waren. Diese lagen in Braunschweig im Normalfall beim Rentenkäufer, der die Hauptsumme in Braunschweig entrichten musste und auch die Auszahlungen hier empfangen sollte. ${ }^{47}$ Allerdings erforderten Kapital- und Zinszahlungen die Anwesenheit des Rentenkäufers nicht unbedingt, sondern konnten auch

47 Ähnliche Einschätzungen gibt es auch für andere Märkte: Rothmann (2007), S. 186; Fuhrmann (2016), S. 20 f.; Albers (1939), S. 49 f. 
durch Bevollmächtigte getätigt werden, nicht selten Bürger der auszahlenden Stadt. ${ }^{48}$ Das relativierte die Kosten, die für den Käufer anfielen. Trotzdem war die Abwicklung der Rentengeschäfte für den Käufer im Vergleich zum Rentenkauf in der Heimatstadt zweifelsohne mit höheren Transaktionskosten verbunden.

Weiterhin müssen höhere Zinssätze auch im Zusammenhang mit Konversionen und Laufzeiten der Renten gesehen werden. Renten wurden gerade bei hohen Ausgangszinssätzen und starker Zinssatzentwicklung nach unten gerne mehrfach auf einen niedrigeren Zinssatz konvertiert, teilweise unter Protest der Käufer. ${ }^{49}$ Alternativ wurden Neurenten zur Auslösung von bestehenden Verträgen mit höheren Zinssätzen verkauft. $^{50}$ Kurze Laufzeiten und Konversionen galten für Braunschweig vor allem für die Zeit von 1541 und 1558, während die 1493 - und in den umliegenden Jahren - verkauften Stadtrenten durchaus lange Laufzeiten haben konnten. Von den 31 Renten, die zwischen 1547 und 1554 zu sechs Prozent verkauft wurden, liegen zu 22 Informationen dazu vor, wie lange der ursprüngliche Zinssatz galt. Bereits nach den im ,Weddeschatbuch' überlieferten Rückkaufsjahren $(22 / 31)$ betrug die durchschnittliche Laufzeit nur gut sechs Jahre; betrachtet man aber neben Rückkauf auch die erste Konversion, so verkürzt sich die durchschnittliche Laufzeit der ursprünglichen Verträge mit sechs Prozent Zinsen auf nur 4,5 Jahre. Eine 1548 verkaufte Rente zu sechs Prozent wurde bis zu ihrer Ablösung 1573 sogar drei Mal nach unten konvertiert, so 1551 auf fünf Prozent, 1559 auf vier Prozent und 1565 auf drei Prozent..$^{51}$ Bei 15 Jahren Vertragslaufzeit lag die durchschnittliche Gültigkeit eines Zinssatzes bei nur 6,25 Jahren. Rentenkäufer mussten also davon ausgehen, dass hohe Zinssätze von Seiten der Stadt baldmöglichst nach unten konvertiert wurden.

Hinzu kommt, dass auch das mit der Geldanlage verbundene Risiko stieg, obgleich städtische Anleihen als vergleichsweise sichere Anlagemöglichkeit gelten dürfen..$^{52} \mathrm{Die}$ auswärtigen Rentenkäufer in Braunschweig legten ihr Kapital in einer Stadt an, deren Haushalt bereits zuvor in schlechter Verfassung gewesen war und für die aufgrund ihrer bestehenden politischen Situation ein relativ hohes Risiko der Nichtleistung von Rentenzahlungen bestand. Für Osnabrück etwa wurde gezeigt, dass deutliche negative Zusammenhänge zwischen Kriegskosten und Rentenzahlungen bestehen: fielen hohe Verteidigungskosten an, dann sanken die Rentenzahlungen..$^{53}$ Zeitweise Zahlungsunfähigkeit von Städten war keine Seltenheit; Auswärtige schlossen oft Vergleiche, um zumindest einen Teil ihrer Zahlungen zu erhalten. ${ }^{54}$ Entsprechend müssen höhere Zins-

Albers (1939), S. 50; Beyer (1901), S. 120 f., 124-126.

Albers (1939), S. 49.

Die im Vergleich zu Einheimischen um 1-2 2/3\% höheren Zinssätze für Straßburger Käufer in Basel 1448/49 wurden in den folgenden Jahren nach unten konvertiert, Gilomen (2003), S. 183. Osnabrück nutzte etwa 1509 nachweislich sein Rückkaufsrecht, wenn Neurenten mit niedrigerem Zinssatz verkauft werden konnten, Eberhardt (1996), S. 49. Zu Konversionen weiterhin bei Knipping (1894), S. 370 f.; Beyer (1901), S. 86 f., 89 f.; Gilomen (2018), S. 74.

Sh. Tabelle 3 im Anhang.

Fuhrmann (2003), S. 13.

Eberhardt (1996), S. 54 .

Idem, S. 46 f.; Rothmann (1998), S. 430-432, 442-448; Beyer (1901), S. 101-106, 109, 111 f., 114. 
sätze auch im Kontext mit zu erwartenden kurz- oder gar längerfristigen Problemen bei der Auszahlung gesehen werden. ${ }^{55}$

Im Lichte unserer Auswertung können die Anlageentscheidungen nicht hauptsächlich aus Zinssatzdifferenzen erklärt werden. So ist Gilomen zuzustimmen, dass mit einem Zinssatzniveau von meist fünf Prozent oder weniger im 15. und 16. Jahrhundert profitorientierte Anlagen nicht mehr häufig gewesen sind. ${ }^{6}$ Höhere Zinssätze können allenfalls als Ausgleich für das Risiko gesehen werden, dass der Käufer mit seiner Kapitalanlage einging.

\section{Kreditbeziehungen und städtische Autonomie}

Wie zuletzt zu zeigen sein wird, spielen politische Gründe eine bedeutende Rolle bei der Mobilisierung auswärtigen Kapitals. Wie wir gesehen haben, sind es vor allem Zeiten auswärtiger Konflikte, die die Zahl der Rentenkäufe in die Höhe schnellen lassen. Betrachten wir exemplarisch die ,Große Stadtfehde‘ von 1492 bis 1494, zu deren Finanzierung Braunschweig mehr Renten verkaufte als jemals zuvor oder danach.

Tabelle 6: Käufergruppen 1493, Beobachtungen zu Kapital in gs.

\begin{tabular}{lcccc}
\hline Käufergruppen & $\mathrm{n}$ & in $\%$ & Kapital in gs & in \% \\
\hline Geistlicher Stand & 38 & 27,3 & 320.243 & 8,3 \\
Körperschaft, geistlich & 21 & 15,1 & 478.397 & 12,4 \\
Körperschaft, städtisch & 24 & 17,3 & 2.155 .935 & 55,8 \\
Privatperson & 56 & 40,3 & 906.752 & 23,5 \\
\hline Gesamtergebnis & 139 & 100,0 & 3.861 .326 & 100,00 \\
\hline
\end{tabular}

Quelle: StA Braunschweig, B I 12, Bd. 3. Grundlage zur Umrechnung in gs: unveröffentlichte Datenzusammenstellung zur Verfügung gestellt durch Oliver Volckart.

Bei den Rentenverkäufen des Jahres 1493 fällt vor allem die hohe Kapitalbeteiligung von städtischen Körperschaften, hier ausschließlich Stadträten, auf. Über 55 Prozent der Kapitalien werden von anderen Ratskollegien aufgebracht (s. Tabelle 6). Fast alle von Räten erworbenen Renten wurden in den Jahren der Stadtfehde gekauft. Die Räte von Königsberg, Danzig, Frankfurt, Hamburg, Lübeck, Lüneburg, Magdeburg und Stralsund erwarben jeweils Renten von 3.00o Rheinischen Gulden und mehr. Bremen kaufte zudem zwei Renten in Höhe von insgesamt 2.00o Rheinischen Gulden. Im Gedenkbuch der Stadt ist darüber hinaus eine Empfangsbestätigung des Braunschweiger Rates über 2.00o Rheinische Gulden von der Stadt Bremen verzeichnet, als Auslösung einer Rente, welche die Stadt Braunschweig in Bremen 1462 gekauft hatte. ${ }^{57}$ Die Renten al- 
leine stellen also nicht die Gesamtheit der in diesem Jahr durch andere Räte verfügbar gemachten Kapitalien dar.

Die von den Räten erworbenen Großrenten und andere bereitgestellte Summen stellen eine politische Unterstützung für die Stadt Braunschweig dar. Der Rentenkauf des 15. und v. a. des 16. Jahrhunderts muss in Zusammenhang gesehen werden mit der politischen Entwicklung der Zeit. Für das 15. Jahrhundert wurde eine zunehmende Politisierung der sächsischen Städtebünde festgestellt, sie wurden zum „Instrument der Bewältigung von Konflikten, die Freiheit und Autonomie der Stadt in immer höherem Maße bedrohten “ ${ }^{\text {(58 }}$. Auch die Hanse nahm im 16. Jahrhundert „immer stärkter den Charakter eines Städtebundes an, der das Ziel der Verteidigung der Freiheit der Hansestädte verfolgte “" ${ }^{99}$ Fast zwei Drittel der Rentenkäufer der 1490er Jahre kommen dann auch aus den führenden Bündnisstädten. Gerade Hildesheim, Braunschweigs wichtigster Geldgeber, zeichnet sich neben Braunschweig selbst durch besonderes Engagement in den sächsischen Städtebünden aus: nur diese beiden Städte haben an allen 28 allgemeinen sächsischen Städtebünden zwischen 1382 und 1490 teilgenommen. ${ }^{60}$ In dem ersten schweren Konflikt mit den Herzögen in den 1490ern kam Hildesheim Braunschweig dann auch „auf hansisches Geheiß“ und mit finanzieller Unterstützung durch die Seestädte zur Hilfe und Braunschweig konnte den Konflikt für sich entscheiden. ${ }^{61}$ Neben Bündnisverträgen erforderte der Erhalt städtischer Autonomie auch Geldmittel, die durch regionale und hansische Bündnispartner bereitgestellt wurden.

Auswärtiges Kapital wurde dabei in Krisenzeiten weniger angeboten denn als politische Unterstützung gezielt angefragt. Bremens Anleihen in Lübeck und Braunschweig etwa sollen schriftliche Bitten um Darlehen vorausgegangen sein und sie werden auf die engen Beziehungen durch die Hansetage zurückgeführt. ${ }^{62}$ Belegt sind weiterhin Rentenverkäufe Bremens zur finanziellen Unterstützung Lübecks. ${ }^{63}$ Auch für Braunschweig ist uns mit dem Brief der Stadt an Köln von 1493 eine solche Anfrage überliefert. Der Brief nennt die Fehde mit dem Herzog als Grund für die Geldnot der Stadt, die nun um Rentenkäufe der Stadt Köln und ihrer Bürger und Einrichtungen bittet. Köln soll „up unse segele unde breve eynen summen goldes up jarlike tynse uns willen laten tonhanden komen", also Braunschweig Gelder bereitstellen, für welche die Käufer Rentenbriefe erhalten sollen - eine bestimmte Summe und der Zinssatz werden hier nicht genannt. Auch soll Köln zugunsten Braunschweigs „by juwen borgern, capitteln, godeshusern unde andern enden dar men goldes bekomen mach", also bei seinen Bürgern, Kirchen, Gotteshäusern und wo man sonst Gold bekommen möge wegen Geldern anfragen. Braunschweig verspricht die Renten jährlich in Köln zu entrichten. ${ }^{64}$ Räte vermittelten also auch Anfragen an weitere potentielle Kapitalgeber innerhalb ihrer Städte - eine Praxis, die ebenso

Puhle (1985), S. 198 (Zitat); Steinführer (2017), S. 21-23.

Steinführer (2017), S. 32 (Zitat).

Puhle (1985), S. 209.

Moderhack (1985), S. $34 \mathrm{f}$.

Albers (1939), S. 43.

Idem, S. 37.

Stadtarchiv Braunschweig, B I 2 Gedenkbücher, Bd. 4: Liber D (1485-1526), S. 133. 
für andere Städte bekannt ist. ${ }^{65}$ Eine Empfangsbestätigung im Gedenkbuch der Stadt aus demselben Jahr spricht dann auch von Geldern, die zur Finanzierung der Stadtfehde durch Hildesheim zugestellt wurden, namentlich 150 Rheinische Gulden von 6 Dörfern im Gericht Seesen und 300 Rheinische Gulden der Stadt Hildesheim. ${ }^{66}$

Zur weiteren Unterstützung Braunschweigs wurden 1515 Vergleiche über Rentenzahlungen mit Rentenkäufern aus Magdeburg und Lübeck zur finanziellen Entlastung der Stadt geschlossen: Renten zahlreicher Magdeburger Bürger, die zur ,Rettung und Erhaltung der Freiheit der Stadt' während der Stadtfehde zu sechs Prozent und in den Folgejahren zu fünf Prozent durch Braunschweig verkauft worden waren, werden für vier Jahre auf vier Prozent herabgesetzt; die Domkirche zu Lübeck verzichtet weiterhin auf 1 1/ 2 Jahre Rente und den fünften Pfennig der Rente diesen Jahres. ${ }^{67}$ Während weder die Stadt Köln noch ihre Bürger und Einrichtungen der Anfrage Braunschweigs gefolgt sind, zeigen die Rentenverkäufe und getroffenen Vergleiche zu Zahlungsverpflichtungen dieser Zeit, dass die oben genannten Stadträte, aber auch Bürger regionaler und hansischer Bündnispartner durch ihre Rentenkäufe die Stadt in ihrer Notlage zu unterstützen suchten. Die auswärtigen Rentenkäufe sind also auch und vor allem durch politische Beihilfe zu erklären, zum Schutz städtischer Autonomie durch Kreditnetzwerke, in Ergänzung regionaler und hansischer Städtebünde.

\section{Ergebnisse}

Zusammenfassend lässt sich feststellen, dass Auswärtige einen bedeutenden Anteil am Braunschweiger Rentenmarkt hatten. Seit dem späten 15. Jahrhundert wurde ungefähr ein Drittel der Stadtrenten an Auswärtige verkauft und also ein nennenswerter Teil des Braunschweiger Kapitalbedarfs außerhalb der Stadt gedeckt. Die meisten Verträge wurden dabei innerhalb der Region abgeschlossen. Die Kreditbeziehungen bewegen sich deutlich entlang der durch den Warenhandel gezeichneten Linien. Sie verdichten sich regional und überregional in wichtigen wirtschaftlichen und politischen Knotenpunkten. Braunschweigs überlokale Rentenverkäufe entsprechen in ihren räumlichen Mustern bekannten Beziehungsnetzwerken der Stadt. Während weltliche Privatpersonen, vor allem die städtische Oberschicht, durchgehend eine wichtige Käufergruppe darstellten, spielten geistliche Personen und Körperschaften nur bis zur Reformation eine bedeutende Rolle als Kapitalgeber. Stadträte waren nur punktuell als Rentenkäufer aktiv, in Notsituationen stellten sie aber kurzfristig größere Summen bereit.

Braunschweig versuchte wie andere Städte, die Rentenverkäufe allgemein auf einem moderaten Level zu halten, um Überschuldung zu vermeiden. Durchschnittlich nur 17

65 Die Vermittlung von Kapitalien verschiedener Käufer soll in solchen Fällen über die Stadträte organisiert worden sein. Dabei wurden private Kapitalien durch die jeweilige Stadtregierung zusammengeführt und geschlossen übergeben. Einzelkäufer agierten also häufig nicht unmittelbar auf den Markt, sondern in Vermittlung durch ihre Heimatstadt, Rothmann (2003), S. 189.

66 Stadtarchiv Braunschweig, B I 2 Gedenkbücher, Bd. 4: Liber D (1485-1526), S. 106.

67 Idem, S. $340 \mathrm{f}$. 
Rentenverkäufe pro Jahr stehen sprunghaften Anstiegen gegenüber, mit einem Höchstwert von 228 verkauften Renten im Jahr 1493. Nach kurzen Phasen stark erhöhter Rentenverkäufe kehren die Verkäufe immer wieder allmählich auf ein niedriges Niveau von höchstens zehn bis 20 verkauften Renten pro Jahr zurück, bis am Ende unseres Untersuchungszeitraums die Verkäufe schließlich ganz ausbleiben. Wie auch für andere Städte festgestellt, waren es vor allem äußere Konflikte, die die Stadt zur Kreditaufnahme zwangen. Der dringende Geldbedarf in Auseinandersetzungen mit den Landesherren ließ Braunschweig gezielt auswärtige Kapitalien nachsuchen. Dabei wurden auswärtigen Rentenkäufern in Zeiten besonders hohen städtischen Kapitalbedarfs höhere Zinssätze angeboten. Man bemühte sich allerdings, diese möglichst bald nach unten zu konvertieren und die Rentensummen abzulösen. Die auch hier mit der Distanz zunehmende Kapitalhöhe ergibt sich aus den politischen Netzwerken der Stadt. Städtische Bündnistätigkeit und städtischer Rentenmarkt müssen dabei in engem Zusammenhang gesehen werden: Renten sind in einer Zeit des Kampfs um städtische Autonomie Instrument gegenseitiger finanzieller Unterstützung in politischen Notlagen. Dies gilt in besonderem Maße für andere Ratskollegien, aber auch für Privatpersonen und geistliche Körperschaften.

Wir konnten in dieser Studie die Vernetzung der Rentenmärkte anhand der Braunschweiger Stadtrentenverkäufe nur in ersten Ansätzen analysieren. Die vorliegende Studie soll dazu einladen die Kapitalmarktvernetzungen der deutschsprachigen Städte weiter zu erforschen, auch im Vergleich mit anderen Regionen. In Zukunft müssen weiteren Studien neben auswärtigen Rentenkäufern und Kapitalnetzwerken auch die institutionellen Grundlagen der Kapitalmärkte näher untersuchen. Für die holländischen Städte liegen hierzu bereits erste Forschungen vor. ${ }^{68}$ Weiterhin müssen die Konditionen und Praktiken des Rentenkaufs vergleichend in den Blick genommen werden. Die Analyse von Verträgen, wie etwa für Basel ansatzweise schon geschehen, ${ }^{69}$ stellt eine wichtige Ergänzung zur quantitativen Auswertung der Marktentwicklung anhand von Zinssätzen und Rentenschuld dar. Auch vermittelnde Instanzen, wie regionale Kapitalmärkte, Messen und Agenten, verdienen eine umfassendere Behandlung. Nur durch die weitere Erforschung auswärtiger Rentenkäufer und der instutitionellen Rahmenbedingungen ihrer Kapitalanlagen können wir die Entwicklung vormoderner Kapitalmärkte umfassend nachvollziehen. 


\section{Anhang}

Tabelle 1: Einheimische und auswärtige Wiederkaufsrentenkäufe nach den Braunschweiger „Weddeschatbüchern“, 1361-1599

\begin{tabular}{|c|c|c|c|c|c|c|c|}
\hline Jahr & $\begin{array}{r}\text { Alle } \\
\text { Käufer }\end{array}$ & $\begin{array}{r}\text { Auswärtige } \\
\text { Käufer }\end{array}$ & in \% & Jahr & $\begin{array}{r}\text { Alle } \\
\text { Käufer }\end{array}$ & $\begin{array}{r}\text { Auswärtige } \\
\text { Käufer }\end{array}$ & in $\%$ \\
\hline 1361 & 1 & & & 1410 & 3 & & \\
\hline 1366 & 1 & & & 1411 & 4 & & \\
\hline 1367 & 2 & & & 1412 & 14 & & \\
\hline 1370 & 5 & & & 1413 & 7 & & \\
\hline 1373 & 1 & & & 1415 & 11 & 1 & 9 \\
\hline 1376 & 2 & & & 1416 & 15 & 1 & 7 \\
\hline 1384 & 1 & & & 1417 & 6 & & \\
\hline 1385 & 1 & & & 1418 & 10 & & \\
\hline 1386 & 1 & & & 1420 & 7 & & \\
\hline 1387 & 2 & & & 1421 & 5 & & \\
\hline 1388 & 3 & & & 1422 & 4 & & \\
\hline 1389 & 1 & & & 1423 & 1 & & \\
\hline 1390 & 1 & 1 & 100 & 1424 & 5 & & \\
\hline 1392 & 5 & 1 & 20 & 1425 & 2 & & \\
\hline 1393 & 6 & 1 & 17 & 1426 & 4 & & \\
\hline 1394 & 3 & & & 1427 & 13 & & \\
\hline 1395 & 11 & & & 1428 & 2 & & \\
\hline 1396 & 9 & & & 1429 & 1 & & \\
\hline 1397 & 12 & 2 & 17 & 1430 & 4 & & \\
\hline 1398 & 13 & 3 & 23 & 1431 & 2 & 1 & 50 \\
\hline 1399 & 8 & & & 1432 & 19 & & \\
\hline 1400 & 9 & 2 & 22 & 1433 & 17 & 5 & 29 \\
\hline 1401 & 5 & & & 1434 & 17 & 5 & 29 \\
\hline 1402 & 3 & & & 1435 & 13 & 2 & 15 \\
\hline 1403 & 7 & & & 1436 & 8 & 1 & 13 \\
\hline 1404 & 3 & & & 1437 & 19 & 4 & 21 \\
\hline 1405 & 2 & & & 1438 & 9 & 1 & 11 \\
\hline 1406 & 3 & & & 1439 & 35 & 3 & 9 \\
\hline 1407 & 2 & & & 1440 & 15 & 2 & 13 \\
\hline 1408 & 2 & & & 1441 & 20 & 1 & 5 \\
\hline 1409 & 3 & & & 1442 & 13 & 1 & 8 \\
\hline
\end{tabular}




\begin{tabular}{|c|c|c|c|c|c|c|c|}
\hline Jahr & $\begin{array}{r}\text { Alle } \\
\text { Käufer }\end{array}$ & $\begin{array}{r}\text { Auswärtige } \\
\text { Käufer }\end{array}$ & in \% & Jahr & $\begin{array}{r}\text { Alle } \\
\text { Käufer }\end{array}$ & $\begin{array}{r}\text { Auswärtige } \\
\text { Käufer }\end{array}$ & in $\%$ \\
\hline 1443 & 8 & & & 1483 & 1 & & \\
\hline 1444 & 13 & 1 & 8 & 1484 & 8 & & \\
\hline 1445 & 8 & 2 & 25 & 1485 & 28 & 1 & 4 \\
\hline 1446 & 18 & 5 & 28 & 1486 & 105 & 15 & 14 \\
\hline 1447 & 41 & 5 & 12 & 1487 & 25 & 2 & 8 \\
\hline 1448 & 41 & 11 & 27 & 1488 & 4 & & \\
\hline 1449 & 40 & 7 & 18 & 1489 & 17 & 2 & 12 \\
\hline 1450 & 32 & 3 & 9 & 1490 & 15 & 5 & 33 \\
\hline 1451 & 30 & 2 & 7 & 1491 & 14 & 4 & 29 \\
\hline 1452 & 31 & 5 & 16 & 1492 & 54 & 21 & 39 \\
\hline 1453 & 35 & & & 1493 & 228 & 141 & 62 \\
\hline 1454 & 20 & 4 & 20 & 1494 & 142 & 59 & 42 \\
\hline 1455 & 5 & 1 & 20 & 1495 & 95 & 42 & 44 \\
\hline \multirow[t]{2}{*}{1456} & 2 & & & 1496 & 81 & 44 & 54 \\
\hline & & & & 1497 & 103 & 45 & 44 \\
\hline 1457 & 60 & 6 & 10 & 1498 & 85 & 47 & 55 \\
\hline 1458 & 4 & & & 1499 & 76 & 40 & 53 \\
\hline 1459 & 20 & 7 & 35 & 1500 & 62 & 20 & 32 \\
\hline 1460 & 13 & 1 & 8 & 1501 & 65 & 7 & 11 \\
\hline 1461 & 3 & & & 1502 & 43 & 15 & 35 \\
\hline 1462 & 4 & 1 & 25 & 1503 & 65 & 23 & 35 \\
\hline 1463 & 5 & & & 1504 & 58 & 20 & 34 \\
\hline 1464 & 1 & & & 1505 & 51 & 23 & 45 \\
\hline 1465 & 1 & & & 1506 & 35 & 13 & 37 \\
\hline 1466 & 6 & 1 & 17 & 1507 & 50 & 14 & 28 \\
\hline 1467 & 18 & 2 & 11 & 1508 & 76 & 31 & 41 \\
\hline \multirow[t]{2}{*}{1468} & 4 & & & 1509 & 62 & 19 & 31 \\
\hline & & & & 1510 & 64 & 11 & 17 \\
\hline 1469 & 2 & & & 1511 & 51 & 14 & 27 \\
\hline 1470 & 6 & 1 & 17 & 1512 & 40 & 8 & 20 \\
\hline 1473 & 1 & & & 1513 & 17 & 8 & 47 \\
\hline 1475 & 2 & 1 & 50 & 1514 & 16 & 2 & 13 \\
\hline 1476 & 1 & & & 1515 & 23 & 2 & 9 \\
\hline 1479 & 1 & & & 1516 & 41 & 11 & 27 \\
\hline 1480 & 1 & & & 1517 & 42 & 16 & 38 \\
\hline
\end{tabular}




\begin{tabular}{|c|c|c|c|c|c|c|c|}
\hline Jahr & $\begin{array}{r}\text { Alle } \\
\text { Käufer }\end{array}$ & $\begin{array}{r}\text { Auswärtige } \\
\text { Käufer }\end{array}$ & in \% & Jahr & $\begin{array}{r}\text { Alle } \\
\text { Käufer }\end{array}$ & $\begin{array}{r}\text { Auswärtige } \\
\text { Käufer }\end{array}$ & in \% \\
\hline 1518 & 31 & 16 & 52 & 1554 & 65 & 19 & 29 \\
\hline 1519 & 48 & 29 & 60 & 1555 & 8 & 1 & 13 \\
\hline 1520 & 37 & 12 & 32 & 1556 & 7 & 3 & 43 \\
\hline 1521 & 32 & 9 & 28 & 1557 & 14 & 8 & 57 \\
\hline 1522 & 34 & 4 & 12 & 1558 & 26 & 14 & 54 \\
\hline 1523 & 32 & 11 & 34 & 1559 & 62 & 23 & 37 \\
\hline 1524 & 32 & 10 & 31 & 1560 & 15 & 6 & 40 \\
\hline 1525 & 24 & 10 & 42 & 1561 & 17 & 2 & 12 \\
\hline 1526 & 17 & 10 & 59 & 1562 & 12 & 3 & 25 \\
\hline 1527 & 7 & & & 1563 & 6 & 3 & 50 \\
\hline 1528 & 13 & 4 & 31 & 1564 & 2 & 1 & 50 \\
\hline 1529 & 16 & 3 & 19 & 1565 & 28 & 11 & 39 \\
\hline 1530 & 27 & 1 & 4 & 1566 & 9 & 2 & 22 \\
\hline 1531 & 21 & 2 & 10 & 1567 & 24 & 7 & 29 \\
\hline 1532 & 16 & 6 & 38 & 1568 & 28 & 6 & 21 \\
\hline 1533 & 10 & 3 & 30 & 1569 & 8 & 1 & 13 \\
\hline 1534 & 14 & 3 & 21 & 1570 & 12 & 4 & 33 \\
\hline 1535 & 20 & 8 & 40 & 1571 & 8 & 2 & 25 \\
\hline 1536 & 11 & 4 & 36 & 1572 & 10 & 1 & 10 \\
\hline 1537 & 12 & 4 & 33 & 1573 & 5 & 1 & 20 \\
\hline 1538 & 7 & & & 1574 & 3 & 1 & 33 \\
\hline 1539 & 17 & 6 & 35 & 1575 & 2 & 1 & 50 \\
\hline 1540 & 7 & 3 & 43 & 1576 & 3 & 1 & 33 \\
\hline 1541 & 3 & 2 & 67 & 1578 & 7 & 4 & 57 \\
\hline 1542 & 5 & & & 1579 & 3 & 2 & 67 \\
\hline 1543 & 5 & & & 1580 & 5 & 2 & 40 \\
\hline 1544 & 3 & & & 1581 & 4 & 4 & 100 \\
\hline 1546 & 1 & & & 1582 & 2 & 1 & 50 \\
\hline 1547 & 13 & 6 & 46 & 1583 & 4 & & \\
\hline 1548 & 64 & 23 & 36 & 1584 & 1 & 1 & 100 \\
\hline 1549 & 24 & 6 & 25 & 1585 & 4 & 1 & 25 \\
\hline 1550 & 22 & 8 & 36 & 1586 & 1 & & \\
\hline 1551 & 65 & 17 & 26 & 1587 & 3 & 1 & 33 \\
\hline 1552 & 46 & 11 & 24 & 1594 & 1 & & \\
\hline 1553 & 57 & 11 & 19 & 1596 & 1 & & \\
\hline
\end{tabular}

Quelle: StA Braunschweig, B I 12, Bde. 1-6. 
Tabelle 2: Herkunftsorte und Kapitalumfang zwischenstädtischer Stadtrentengeschäfte in Braunschweig bis 1599, nach Anzahl der verkauften Renten

\begin{tabular}{|c|c|c|c|c|c|c|}
\hline Herkunftsorte & $\begin{array}{r}\text { Entfernung } \\
\text { in } \mathbf{~ k m}\end{array}$ & n, gesamt & in $\%$ & $\begin{array}{r}\mathbf{n}, \text { Kapital } \\
\text { in gs }\end{array}$ & $\begin{array}{r}\text { Kapital } \\
\text { in gs }\end{array}$ & in $\%$ \\
\hline Hildesheim & 41 & 296 & 23,1 & 281 & 2.507 .079 & 17,2 \\
\hline Goslar & 41 & 117 & 9,1 & 103 & 1.538 .735 & 10,6 \\
\hline Magdeburg & 77 & 115 & 9,0 & 112 & 2.278 .320 & 15,7 \\
\hline Halberstadt & 55 & 78 & 6,1 & 61 & 677.773 & 4,7 \\
\hline Celle & 50 & 54 & 4,2 & 33 & 331.319 & 2,3 \\
\hline Lübeck & 178 & 37 & 2,9 & 27 & 1.012 .225 & 7,0 \\
\hline Wienhausen & 41 & 32 & 2,5 & 22 & 83.878 & 0,6 \\
\hline Peine & 20 & 29 & 2,3 & 28 & 165.956 & 1,1 \\
\hline Helmstedt & 33 & 26 & 2,0 & 18 & 109.710 & 0,8 \\
\hline Wöltingerode & 34 & 19 & 1,5 & 15 & 343.507 & 2,4 \\
\hline Bremen & 147 & 18 & 1,4 & 4 & 77.257 & 0,5 \\
\hline Derneburg & 34 & 15 & 1,2 & 13 & 62.787 & 0,4 \\
\hline Frankfurt am Main & 272 & 15 & 1,2 & 13 & $365 \cdot 318$ & 2,5 \\
\hline Lüneburg & 109 & 14 & 1,1 & 11 & $735 \cdot 734$ & 5,1 \\
\hline Salzgitter-Steterburg & 9 & 14 & 1,1 & 12 & 36.114 & 0,2 \\
\hline Badeleben & 43 & 12 & 0,9 & 10 & 103.096 & 0,7 \\
\hline Dorstadt & 19 & 12 & 0,9 & 10 & 109.873 & 0,8 \\
\hline Lüttgenrode & 35 & 12 & 0,9 & 8 & 25.021 & 0,2 \\
\hline Einbeck & 67 & 11 & 0,9 & 11 & 85.680 & 0,6 \\
\hline Hamersleben & 45 & 11 & 0,9 & 9 & 129.255 & 0,9 \\
\hline Querum & 4 & 11 & 0,9 & 10 & 18.684 & 0,1 \\
\hline Schöningen & 33 & 11 & 0,9 & 5 & 27.163 & 0,2 \\
\hline Erfurt & 147 & 10 & 0,8 & 2 & 36.727 & 0,3 \\
\hline Bad Gandersheim & 56 & 10 & 0,8 & 9 & 98.169 & 0,7 \\
\hline Huysburg & 48 & 10 & 0,8 & 9 & 311.185 & 2,1 \\
\hline Salzwedel & 78 & 10 & 0,8 & 9 & 138.050 & 0,9 \\
\hline Eddesse & 26 & 9 & 0,7 & 8 & 102.238 & 0,7 \\
\hline Oebisfelde & 36 & 9 & 0,7 & 4 & 56.566 & 0,4 \\
\hline Hamburg & 147 & 8 & 0,6 & 8 & 869.957 & 6,0 \\
\hline Heiningen, Helmstedt & 22 & 8 & 0,6 & 8 & 53.778 & 0,4 \\
\hline Hankensbüttel & 51 & 6 & 0,5 & 4 & 32.303 & 0,2 \\
\hline Hondelage & 7 & 6 & 0,5 & 6 & 29.812 & 0,2 \\
\hline Hornburg & 27 & 6 & 0,5 & 2 & 46.778 & 0,3 \\
\hline Huysburg & 47 & 6 & 0,5 & 5 & 48.073 & 0,3 \\
\hline
\end{tabular}




\begin{tabular}{|c|c|c|c|c|c|c|}
\hline Herkunftsorte & $\begin{array}{r}\text { Entfernung } \\
\text { in } \mathbf{k m}\end{array}$ & n, gesamt & in $\%$ & $\begin{array}{r}\text { n, Kapital } \\
\text { in gs }\end{array}$ & $\begin{array}{r}\text { Kapital } \\
\text { in gs }\end{array}$ & in \% \\
\hline Verden & 114 & 6 & 0,5 & 6 & 105.611 & 0,7 \\
\hline Wittenburg, Elze & 58 & 6 & 0,5 & 6 & 39.987 & 0,3 \\
\hline Göttingen & 91 & 5 & 0,4 & 3 & 37.787 & 0,3 \\
\hline Seesen & 48 & 5 & 0,4 & & & \\
\hline Uetze & 31 & 5 & 0,4 & 4 & 47.170 & 0,3 \\
\hline Wittstock & 165 & 5 & 0,4 & 2 & 18.736 & 0,1 \\
\hline Wolfenbüttel & 12 & 5 & 0,4 & & & \\
\hline Arnsberg & 195 & 4 & 0,3 & 4 & 135.149 & 0,9 \\
\hline Bad Münder & 74 & 4 & 0,3 & 4 & 12.735 & 0,1 \\
\hline Bienrode & 6 & 4 & 0,3 & 4 & 51.924 & 0,4 \\
\hline Denstorf & 8 & 4 & 0,3 & 4 & 32.145 & 0,2 \\
\hline Marienborn, Sommersdorf & 40 & 4 & 0,3 & 4 & 27.985 & 0,2 \\
\hline Möllenbeck, Rinteln & 102 & 4 & 0,3 & 4 & 80.086 & 0,6 \\
\hline Warle & 26 & 4 & 0,3 & 4 & & \\
\hline Wasbüttel & 17 & 4 & 0,3 & 1 & 5.964 & 0,0 \\
\hline Wendeburg & 11 & 4 & 0,3 & 2 & & \\
\hline Ausleben & 45 & 3 & 0,2 & 2 & 9.846 & 0,1 \\
\hline Beedenbostel & 45 & 3 & 0,2 & 2 & 9.918 & 0,1 \\
\hline Ebstorf & 85 & 3 & 0,2 & 1 & & \\
\hline Hadmersleben & 61 & 3 & 0,2 & 3 & 12.821 & 0,1 \\
\hline Hameln & 81 & 3 & 0,2 & 3 & 37.456 & 0,3 \\
\hline Hannover & 55 & 3 & 0,2 & 2 & 5.706 & 0,0 \\
\hline Heiligendorf & 19 & 3 & 0,2 & & & \\
\hline Loccum & 96 & 3 & 0,2 & 3 & 62.899 & 0,4 \\
\hline Rüningen & 5 & 3 & 0,2 & 3 & 4.572 & 0,0 \\
\hline Salzgitter & 19 & 3 & 0,2 & 3 & 17.553 & 0,1 \\
\hline Stederdorf & 72 & 3 & 0,2 & 3 & 5.793 & 0,0 \\
\hline Alfeld (Leine) & 57 & 2 & 0,2 & 1 & 3.147 & 0,0 \\
\hline Aschersleben & 85 & 2 & 0,2 & 1 & 15.552 & 0,1 \\
\hline Bahrdorf & 35 & 2 & 0,2 & 2 & 14.618 & 0,1 \\
\hline Dahrenhorst, Uetze & 34 & 2 & 0,2 & 1 & 5.964 & 0,0 \\
\hline Danzig & 586 & 2 & 0,2 & 2 & 188.841 & 1,3 \\
\hline Edemissen & 22 & 2 & 0,2 & 1 & 3.531 & 0,0 \\
\hline Escherde & 48 & 2 & 0,2 & 1 & 3.144 & 0,0 \\
\hline Gifhorn & 24 & 2 & 0,2 & 2 & 11.884 & 0,1 \\
\hline Halle an der Saale & 131 & 2 & 0,2 & 2 & 17.843 & 0,1 \\
\hline
\end{tabular}




\begin{tabular}{|c|c|c|c|c|c|c|}
\hline Herkunftsorte & $\begin{array}{r}\text { Entfernung } \\
\text { in km }\end{array}$ & n, gesamt & in $\%$ & $\begin{array}{r}\text { n, Kapital } \\
\text { in gs }\end{array}$ & $\begin{array}{r}\text { Kapital } \\
\text { in gs }\end{array}$ & in $\%$ \\
\hline Höckelheim & 74 & 2 & 0,2 & 2 & 31.659 & 0,2 \\
\hline Kissenbrück & 18 & 2 & 0,2 & 2 & 5.655 & 0,0 \\
\hline Lamme & 6 & 2 & 0,2 & 2 & 5.901 & 0,0 \\
\hline Lamspringe & 49 & 2 & 0,2 & 2 & $5 \cdot 744$ & 0,0 \\
\hline Lüchow, Schloß & 88 & 2 & 0,2 & 2 & 63.422 & 0,4 \\
\hline Meinersen & 25 & 2 & 0,2 & & & \\
\hline Northeim & 73 & 2 & 0,2 & 2 & 31.709 & 0,2 \\
\hline Osterode & 63 & 2 & 0,2 & 2 & 5.961 & 0,0 \\
\hline Quedlinburg & 68 & 2 & 0,2 & 2 & 11.701 & 0,1 \\
\hline Schwalenberg & 101 & 2 & 0,2 & & & \\
\hline Sonnenberg, Vechelde & 13 & 2 & 0,2 & 2 & 5.531 & 0,0 \\
\hline Stendal & 97 & 2 & 0,2 & 2 & 18.975 & 0,1 \\
\hline Völkenrode & 7 & 2 & 0,2 & 1 & 731 & 0,0 \\
\hline Walkenried & 76 & 2 & 0,2 & 2 & 66.375 & 0,5 \\
\hline Wasserleben & 42 & 2 & 0,2 & 2 & 12.104 & 0,1 \\
\hline Werningerode & 51 & 2 & 0,2 & 2 & $7 \cdot 381$ & 0,1 \\
\hline Adensen & 55 & 1 & 0,1 & 1 & 1.431 & 0,0 \\
\hline Adenstedt & 25 & 1 & 0,1 & 1 & 9.533 & 0,1 \\
\hline Astenbeck, Holle & 33 & 1 & 0,1 & 1 & 5.688 & 0,0 \\
\hline Bad Oldesloe & 171 & 1 & 0,1 & 1 & 12.576 & 0,1 \\
\hline Bardowick & 114 & 1 & 0,1 & 1 & 47.210 & 0,3 \\
\hline Berklingen & 22 & 1 & 0,1 & 1 & 877 & 0,0 \\
\hline Bockenem & 39 & 1 & 0,1 & 1 & 6.288 & 0,0 \\
\hline Böddeken & 147 & 1 & 0,1 & 1 & 42.138 & 0,3 \\
\hline Bodenburg & 45 & 1 & 0,1 & 1 & 5.779 & 0,0 \\
\hline Bothmer & 79 & 1 & 0,1 & 1 & 18.304 & 0,1 \\
\hline Burgdorf & 41 & 1 & 0,1 & 1 & 25.449 & 0,2 \\
\hline Dachtmissen, Burgdorf & 39 & 1 & 0,1 & 1 & 3.144 & 0,0 \\
\hline Dahlem & 188 & 1 & 0,1 & 1 & 29.097 & 0,2 \\
\hline Drübeck, Ilsenburg & 47 & 1 & 0,1 & 1 & 5.879 & 0,0 \\
\hline Eldagsen & 60 & 1 & 0,1 & 1 & 9.432 & 0,1 \\
\hline Erkerode & 14 & 1 & 0,1 & 1 & & \\
\hline Fallersleben & 21 & 1 & 0,1 & 1 & 3.121 & 0,0 \\
\hline \multicolumn{7}{|l|}{ Fallersleben, Schloß } \\
\hline Gadenstedt & 21 & 1 & 0,1 & 1 & & \\
\hline Groß Hegesdorf, Apelern & 83 & 1 & 0,1 & 1 & 3.178 & 0,0 \\
\hline
\end{tabular}




\begin{tabular}{lrrrrrr}
\hline Herkunftsorte & $\begin{array}{r}\text { Entfernung } \\
\text { in km }\end{array}$ & n, gesamt & in \% & $\begin{array}{r}\text { n, Kapital } \\
\text { in gs }\end{array}$ & $\begin{array}{r}\text { Kapital } \\
\text { in gs }\end{array}$ & in \% \\
\hline Ilsenburg & 46 & 1 & 0,1 & 1 & 34.202 & 0,2 \\
Königsberg & 714 & 1 & 0,1 & 1 & 94.421 & 0,6 \\
Mühlhausen & 118 & 1 & 0,1 & 1 & 5.688 & 0,0 \\
Schöppenstedt & 22 & 1 & 0,1 & 1 & & \\
Sierße, Vechelde & 13 & 1 & 0,1 & 1 & 8.658 & 0,1 \\
Stralsund & 283 & 1 & 0,1 & 1 & 94.421 & 0,6 \\
Walsrode & 91 & 1 & 0,1 & 1 & & \\
Wismar & 191 & 1 & 0,1 & 1 & 2.966 & 0,0 \\
Wunstorf & 75 & 1 & 0,1 & 1 & 4.402 & 0,0 \\
NN & - & 37 & 2,9 & 23 & 118.699 & 0,8
\end{tabular}

Quelle: StA Braunschweig, B I 12, Bde. 1-6. Grundlage der Umrechnung in gs: unveröffentlichte Datenzusammenstellung von Oliver Volckart.

Tabelle 3: Laufzeiten und Konversionen von Braunschweiger Stadtrentenverträgen zu $6 \%$, 1547-1554

\begin{tabular}{|c|c|c|c|c|c|c|c|c|c|c|}
\hline $\begin{array}{c}\text { Verkauf } \\
\text { zu } 6 \%\end{array}$ & $\begin{array}{c}\text { Konv. } \\
5 \%\end{array}$ & $\begin{array}{c}\text { Konv. } \\
4 \%\end{array}$ & $\begin{array}{c}\text { Konv. } \\
\mathbf{3} \%\end{array}$ & $\begin{array}{c}\text { Rück- } \\
\text { kauf }\end{array}$ & $\begin{array}{c}\text { Lauf- } \\
\text { zeit } \\
6 \%\end{array}$ & $\begin{array}{c}\text { Lauf- } \\
\text { zeit } \\
5 \%\end{array}$ & $\begin{array}{c}\text { Lauf- } \\
\text { zeit } \\
4 \%\end{array}$ & $\begin{array}{c}\text { Lauf- } \\
\text { zeit } \\
3 \%\end{array}$ & $\begin{array}{l}\text { Gesamt- } \\
\text { laufzeit }\end{array}$ & $\begin{array}{c}\text { Durchschn. } \\
\text { Laufzeit pro } \\
\text { Zinssatz }\end{array}$ \\
\hline 1547 & & & & 1551 & 4 & & & & 4 & 4 \\
\hline 1547 & & & & 1556 & 9 & & & & 9 & 9 \\
\hline 1547 & & & & 1556 & 9 & & & & 9 & 9 \\
\hline 1548 & 1551 & 1559 & 1565 & 1573 & 3 & 8 & 6 & & 25 & 8 \\
\hline 1548 & 1552 & & & 1559 & 4 & & & & 11 & 7 \\
\hline 1548 & 1554 & & & & 6 & & & & $\mathrm{nn}$ & \\
\hline 1548 & 1554 & & & & 6 & & & & $\mathrm{nn}$ & \\
\hline 1548 & & 1559 & & 1565 & 11 & 11 & & & 17 & 6 \\
\hline 1548 & & & & & & & & & $\mathrm{nn}$ & \\
\hline 1548 & & & & 1554 & 6 & & & & 6 & 6 \\
\hline 1548 & & & & & & & & & $\mathrm{nn}$ & \\
\hline 1548 & & & & & & & & & $\mathrm{nn}$ & \\
\hline 1548 & & & & 1562 & 14 & & & & 14 & 14 \\
\hline 1548 & & & & & & & & & $\mathrm{nn}$ & \\
\hline 1548 & & & & 1551 & 3 & & & & 3 & 3 \\
\hline 1548 & & & & 1551 & 3 & & & & 3 & 3 \\
\hline 1548 & & & & 1551 & 3 & & & & 3 & 3 \\
\hline 1548 & & & & 1551 & 3 & & & & 3 & 3 \\
\hline
\end{tabular}




\begin{tabular}{|c|c|c|c|c|c|c|c|c|c|c|}
\hline $\begin{array}{c}\text { Verkauf } \\
\text { zu } 6 \%\end{array}$ & $\begin{array}{c}\text { Konv. } \\
5 \%\end{array}$ & $\begin{array}{c}\text { Konv. } \\
4 \%\end{array}$ & $\begin{array}{c}\text { Konv. } \\
3 \%\end{array}$ & $\begin{array}{l}\text { Rück- } \\
\text { kauf }\end{array}$ & $\begin{array}{c}\text { Lauf- } \\
\text { zeit } \\
6 \%\end{array}$ & $\begin{array}{c}\text { Lauf- } \\
\text { zeit } \\
5 \%\end{array}$ & $\begin{array}{c}\text { Lauf- } \\
\text { zeit } \\
4 \%\end{array}$ & $\begin{array}{c}\text { Lauf- } \\
\text { zeit } \\
3 \%\end{array}$ & $\begin{array}{l}\text { Gesamt- } \\
\text { laufzeit }\end{array}$ & $\begin{array}{c}\text { Durchschn. } \\
\text { Laufzeit pro } \\
\text { Zinssatz }\end{array}$ \\
\hline 1548 & & & & 1551 & 3 & & & & 3 & 3 \\
\hline 1548 & & & & 1551 & 3 & & & & 3 & 3 \\
\hline 1548 & & & & 1551 & 3 & & & & 3 & 3 \\
\hline 1549 & 1551 & & & & 2 & & & & $\mathrm{nn}$ & \\
\hline 1549 & 1552 & & & & 3 & & & & $\mathrm{nn}$ & \\
\hline 1549 & & & & 1551 & 2 & & & & 2 & 2 \\
\hline 1549 & & & & 1551 & 2 & & & & 2 & 2 \\
\hline 1550 & & & & 1552 & 2 & & & & 2 & 2 \\
\hline 1551 & 1551 & 1551 & 1565 & & 0 & o & 14 & & $\mathrm{nn}$ & \\
\hline 1553 & $\mathrm{nn}$ & & & & & & & & $\mathrm{nn}$ & \\
\hline 1553 & & & & 1554 & 1 & & & & 1 & 1 \\
\hline 1554 & 1556 & & & & 2 & & & & $\mathrm{nn}$ & \\
\hline 1554 & & & & 1554 & 0 & & & & 0 & 0 \\
\hline
\end{tabular}

Quelle: StA Braunschweig, B I 12, Bde. 5-6.

Tabelle 4: Rentenkäufer mit Aktivitäten in Braunschweig und anderen Märkten für Stadtrenten

\begin{tabular}{|c|c|c|c|c|c|c|c|c|c|c|}
\hline Kategorie & Name & 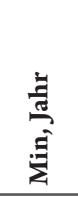 & 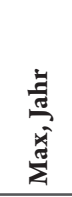 & 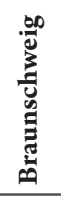 & 苞 & 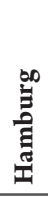 & 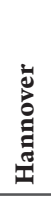 & 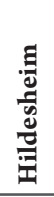 & 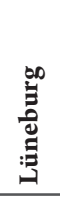 & 节 \\
\hline Körperschaft & & 1385 & 1587 & 147 & 2 & 4 & 26 & 10 & 35 & 224 \\
\hline geistlich & $n 22$ & 1398 & 1587 & 121 & & 2 & 26 & 8 & 28 & 185 \\
\hline Bardowick & Hospital Bardowick & 1449 & 1493 & 2 & & & & & 2 & 4 \\
\hline \multirow[t]{2}{*}{ Goslar } & Kirche SS Cosme \& Damiani & 1403 & 1498 & 4 & & & 1 & & & 5 \\
\hline & Kirche SS Simon \& Judae & 1398 & 1503 & 1 & & & 2 & & & 3 \\
\hline Göttingen & Kloster St, Annen & 1521 & 1564 & 1 & & & & 1 & & 2 \\
\hline \multirow[t]{6}{*}{ Hildesheim } & Domkirche & 1399 & 1502 & 4 & & & 4 & & 2 & 10 \\
\hline & $\begin{array}{l}\text { Kapitel der Kirche Hildes- } \\
\text { heim }\end{array}$ & 1493 & 1508 & 7 & & & & & & 7 \\
\hline & Kirche St, Andreas & 1409 & 1523 & 9 & & & 2 & & 3 & 14 \\
\hline & Kirche St, Crucis & 1420 & 1547 & 7 & & & 3 & & 2 & 12 \\
\hline & Kirche St, Maurici & 1444 & 1524 & 9 & & & 2 & & 3 & 4 \\
\hline & Schwesternkloster & 1493 & 1497 & 6 & & & & & & 6 \\
\hline
\end{tabular}




\begin{tabular}{|c|c|c|c|c|c|c|c|c|c|c|}
\hline Kategorie & Name & 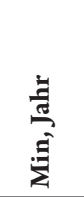 & 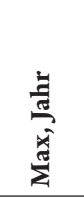 & 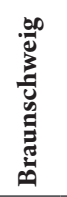 & 节 & 范 & 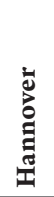 & 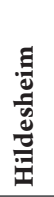 & : & 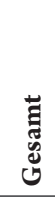 \\
\hline & St. Marien Karthäuserkloster & 1400 & 1583 & 1 & & 1 & 6 & & 3 & 11 \\
\hline Huysburg & Kloster Adersleve & 1523 & 1561 & 5 & & 1 & & & & 6 \\
\hline Lamspringe & Kloster Lamspringe & 1509 & 1512 & 2 & & & & 5 & & 7 \\
\hline Loccum & Kloster Loccum & 1494 & 1498 & 3 & & & 1 & & & 4 \\
\hline Lübeck & Kirche Unsere Liebe Frau & 1493 & 1497 & 2 & & & & & 2 & 4 \\
\hline Magdeburg & Domkirche & 1450 & 1498 & 3 & & & & & 1 & 4 \\
\hline Möllenbeck & Kloster Möllenbeck & 1497 & 1587 & 4 & & & 1 & & & 5 \\
\hline Verden & Kapitel der Kirche Verden & 1481 & 1512 & 9 & & & & & 6 & 15 \\
\hline Walkenried & Kloster Walkenried & 1441 & 1499 & 2 & & & & & 2 & 4 \\
\hline Wienhausen & Kloster Wienhausen & 1438 & 1555 & 17 & & & 2 & & 1 & 20 \\
\hline $\begin{array}{l}\text { Wittenburg, } \\
\text { Elze }\end{array}$ & Kloster Wittenburg & 1494 & 1521 & 5 & & & 2 & & & 7 \\
\hline Wöltingerode & Kloster Wöltingerode & 1433 & 1550 & 18 & & & & 2 & 1 & 21 \\
\hline weltlich & n6 & 1385 & 1560 & 26 & 2 & 2 & & 2 & 7 & 39 \\
\hline Celle & Rat Celle & 1548 & 1560 & 2 & & 1 & & & & 3 \\
\hline Hamburg & Rat Hamburg & 1492 & 1522 & 8 & & & & 1 & & 9 \\
\hline Lübeck & Rat Lübeck & 1385 & 1499 & 4 & 2 & 1 & & & 4 & 11 \\
\hline \multirow[t]{2}{*}{ Magdeburg } & Rat Magdeburg & 1493 & 1523 & 1 & & & & 1 & & 2 \\
\hline & Rat Magdeburg, Altstadt & 1493 & 1505 & 9 & & & & & & 9 \\
\hline Salzwedel & Großer Kaland Salzwedel & 1444 & 1512 & 2 & & & & & 3 & 5 \\
\hline Person & & 1403 & 1606 & 64 & & 14 & 15 & 2 & 17 & 112 \\
\hline geistlich & $n 10$ & 1433 & 1527 & 34 & & & 12 & 1 & 8 & 55 \\
\hline Goslar & Keseberg, Dietrich & 1493 & 1500 & 3 & & & & 1 & & 4 \\
\hline Halberstadt & Quirre, Ludolf & 1455 & 1460 & 2 & & & 1 & & & 3 \\
\hline \multirow[t]{8}{*}{ Hildesheim } & Bringkman, Johann & 1491 & 1500 & 1 & & & 2 & & & 3 \\
\hline & Horn, Sivert & 1494 & 1494 & 1 & & & 1 & & & 2 \\
\hline & Klot, Heinrich & 1494 & 1500 & 2 & & & 1 & & & 3 \\
\hline & Lerte, Johann & 1439 & 1475 & 2 & & & 4 & & 2 & 8 \\
\hline & Pael, Eberhard & 1491 & 1499 & 4 & & & 2 & & & 6 \\
\hline & Steinhof, Borchard & 1433 & 1442 & 1 & & & & & 2 & 3 \\
\hline & Suring, Ludolf & 1493 & 1524 & 13 & & & 1 & & & 14 \\
\hline & $\begin{array}{r}\text { This material is under copyright. } \\
\text { of copyright law is il } \\
\text { This applies in particulart } \\
\text { as well as storage and } \\
\text { O Franz Steine }\end{array}$ & $\begin{array}{l}\text { ny use or } \\
\text { gaal and : } \\
\text { copies, } t \\
\text { rocessing } \\
\text { Verlag, } s\end{array}$ & $\begin{array}{l}\text { side of } t \\
\text { ay be pr } \\
\text { inslatior } \\
\text { n electro } \\
\text { uttgart } 2\end{array}$ & $\begin{array}{l}\text { sec } \\
\text { mi } \\
\text { ics }\end{array}$ & Iming & & & & & \\
\hline
\end{tabular}




\begin{tabular}{|c|c|c|c|c|c|c|c|c|c|c|}
\hline Kategorie & Name & 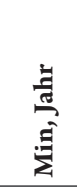 & 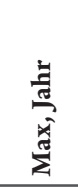 & 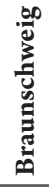 & : & 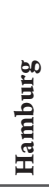 & 离 & 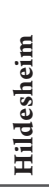 & 先 & 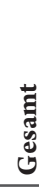 \\
\hline & Verdeman, Ludolf & 1490 & 1507 & 5 & & & & & 4 & 9 \\
\hline weltlich & $n 14$ & 1403 & 1606 & 30 & & 14 & 3 & 1 & 9 & 57 \\
\hline \multirow[t]{8}{*}{ Celle } & Brauwer, Hans & 1568 & 1575 & 1 & & 1 & & & & 2 \\
\hline & Eltze, Eggeling & 1555 & 1564 & 1 & & 4 & & & & 5 \\
\hline & Hollemann, Franz & 1553 & 1560 & 1 & & 1 & & & & 2 \\
\hline & Hollemann, Henning & 1549 & 1563 & 5 & & 2 & & & & 7 \\
\hline & Hollemann, Kord & 1548 & 1559 & 5 & & & 1 & & & 6 \\
\hline & Schulte, Anna & 1548 & 1554 & 3 & & & & & 1 & 4 \\
\hline & Stockman, Heinrich & 1564 & 1571 & 3 & & 4 & & & & 7 \\
\hline & Wolter, Dietrich & 1549 & 1559 & 5 & & & 1 & & & 6 \\
\hline \multirow[t]{2}{*}{ Einbeck } & Raven, Dietrich & 1493 & 1494 & 1 & & & & & 3 & 4 \\
\hline & Raven, Johann & 1494 & 1500 & 1 & & & & & 2 & 3 \\
\hline \multirow[t]{2}{*}{ Goslar } & Detmar, Henning & 1508 & 1536 & 2 & & & & 1 & & 3 \\
\hline & Himteken, Heinrich & 1403 & 1431 & 1 & & & 1 & & & 2 \\
\hline Hildesheim & Damme, Heinrich & 1439 & 1442 & 1 & & & & & 1 & 2 \\
\hline Wolfenbüttel & Herzogin Clara von BS-LB & 1550 & 1606 & & & 2 & & & 2 & 4 \\
\hline
\end{tabular}

Quellen: Braunschweig: Stadtarchiv Braunschweig, B I 12 Weddeschatbücher, Bde. 1-6; Bremen: ${ }^{70}$ Staatsarchiv Bremen, 1-Bs Städtische Urkunden: Schuldverschreibungen; Albers (1939), S. 109-163; Hamburg: Staatsarchiv Hamburg, 311-1 I Kämmerei I, Vol. 2: Urkunden 1496-1866 (Unbefristete Kammerbriefe); Koppmann (1869-1894); Hannover: Stadtarchiv Hannover, Neue Abteilung Bücher, 8242 Stadtobligationsbuch 1387-1533; Urkunden Abteilung 3: Schuldurkunden des Rates (1351-1716); Hildesheim: Stadtarchiv Hildesheim, Bestand 1 Nr. 1275: Schuldverschreibungen; Bestand 50 Nr. 159: Kämmereirechnungen; Lüneburg: Stadtarchiv Lüneburg, Urkunden-Abteilung; AB 51: Darlehensregister (1368-1416); AB 55: Kopie von Rentenbriefen (1441-1492); AB 60: Abschriften von Schuldverschreibungen des Rates zu Lüneburg; AB 65: Schuldurkunden des Rates mit alphabetischem Inhaltsverzeichnis (1492-1516); AB 68: Registrum der Renthe bynnen unde buthen Lüneburg, de me hyr bynnen betaleth (15. \& 1. Drittel 16. Jh.); AB 69: Einnahmen und Ausgaben sowie Rentenbriefe (1534/1565, 1566, 1586); AB 71: Kopien von Verschreibungen des Rates aus der Zeit von 1566 bis 1616; AB 80: Schuldbuch mit Ratsrentenverschreibungen.

70 Wir danken Herrn Ernst Schütz für die Zurverfügungstellung seiner Auswertungen zum Bremer Rentenmarkt. 
Tabelle 5: Herkunft und Gruppen von Rentenkäufern auf dem Braunschweiger Rentenmarkt im Jahr 1493, Höhe des Kapitals in Rheinischen Gulden (RG)

\begin{tabular}{|c|c|c|c|c|c|}
\hline \multirow{2}{*}{$\begin{array}{c}\text { Herkunft Renten- } \\
\text { käufer }\end{array}$} & \multicolumn{2}{|c|}{$\begin{array}{c}\text { Kapital geistlich, } \\
\text { in RG }\end{array}$} & \multicolumn{2}{|c|}{$\begin{array}{c}\text { Kapital weltlich, } \\
\text { in RG }\end{array}$} & \multirow{2}{*}{$\begin{array}{c}\text { Gesamtergebnis } \\
\text { in RG }\end{array}$} \\
\hline & $\begin{array}{l}\text { Einzel- } \\
\text { personen }\end{array}$ & $\begin{array}{c}\text { Körper- } \\
\text { schaf- } \\
\text { ten }\end{array}$ & $\begin{array}{l}\text { Einzel- } \\
\text { personen }\end{array}$ & $\begin{array}{c}\text { Körper- } \\
\text { schaf- } \\
\text { ten } \\
\end{array}$ & \\
\hline Magdeburg & 885 & 3.300 & 24.500 & $5 \cdot 390$ & 34.075 \\
\hline Lübeck & & 1.600 & 5.000 & 8.000 & 14.600 \\
\hline Hamburg & & & 14.000 & & 14.000 \\
\hline Hildesheim & 5.650 & 800 & & 5.120 & 11.570 \\
\hline Frankfurt am Main & & & 6.000 & 4.800 & 10.800 \\
\hline Lüneburg & & 5.500 & 5.000 & & 10.500 \\
\hline Goslar & 300 & 1.800 & & 4.200 & 6.300 \\
\hline Danzig & & & 6.000 & & 6.000 \\
\hline Halberstadt & 2.440 & 500 & & 2.100 & 5.040 \\
\hline Stralsund & & & 3.000 & & 3.000 \\
\hline Königsberg & & & 3.000 & & 3.000 \\
\hline Salzwedel & & & & 2.400 & 2.400 \\
\hline Bremen & & & 2.000 & & 2.000 \\
\hline Bardowick & & 1.500 & & & 1.500 \\
\hline Gandersheim & & & & 1.300 & 1.300 \\
\hline Wittstock & & & & 800 & 800 \\
\hline Einbeck & 400 & & & 300 & 700 \\
\hline Dorstadt & & 600 & & & 600 \\
\hline Huysburg & & 600 & & & 600 \\
\hline Peine & & & & 400 & 400 \\
\hline Wöltingerode & & 400 & & & 400 \\
\hline Stendal & 300 & & & & 300 \\
\hline Derneburg & 100 & & & 100 & 200 \\
\hline Halle an der Saale & & & & 200 & 200 \\
\hline Helmstedt & & 200 & & & 200 \\
\hline Alfeld (Leine) & & & & 100 & 100 \\
\hline $\begin{array}{l}\text { Salzgitter- } \\
\text { Steterburg }\end{array}$ & 100 & & & & 100 \\
\hline Salzgitter & & & & 100 & 100 \\
\hline $\mathrm{nn}$ & & & & 100 & 100 \\
\hline Gesamtergebnis & 10.175 & 16.800 & 68.500 & 35.410 & 130.885 \\
\hline
\end{tabular}

Quelle: StA Braunschweig B I 12, Bd. 3. 


\title{
Quellen- und Literaturverzeichnis
}

\author{
Archivquellen
}

Archiv der Hansestadt Lübeck, ASA Externa Hanseatica, Nrn. 203-204, 207, 209, 211, 213, 218, 224a, $230,235-237,247$.

Staatsarchiv Bremen, 1-Bs Städtische Urkunden: Schuldverschreibungen.

Stadtarchiv Braunschweig, B I 12 Weddeschatbücher, Bde. 1-6.

Stadtarchiv Braunschweig, B I 2 Gedenkbücher, Bd. 4: Liber D (1485-1526).

Stadtarchiv Braunschweig, B III 4:22 Bd. 1, 4:34, 4:45.

Staatsarchiv Hamburg, 311-1 I Kämmerei I, Vol. 2: Urkunden 1496-1866 (Unbefristete Kammerbriefe).

Stadtarchiv Hannover, Neue Abteilung Bücher, 8242 Stadtobligationsbuch 1387-1533.

Stadtarchiv Hannover, Urkunden Abteilung 3: Schuldurkunden des Rates (1351-1716).

Stadtarchiv Hildesheim, Bestand $1 \mathrm{Nr}$, 1275: Schuldverschreibungen.

Stadtarchiv Hildesheim, Bestand 50 Nr, 159: Kämmereirechnungen.

Stadtarchiv Lüneburg, Urkunden-Abteilung; AB 51: Darlehensregister (1368-1416).

Stadtarchiv Lüneburg, AB 55: Kopie von Rentenbriefen (1441-1492).

Stadtarchiv Lüneburg, $\mathrm{AB}$ 60: Abschriften von Schuldverschreibungen des Rates zu Lüneburg.

Stadtarchiv Lüneburg, AB 65: Schuldurkunden des Rates mit alphabetischem Inhaltsverzeichnis (1492-1516); AB 68: Registrum der Renthe bynnen unde buthen Lüneburg, de me hyr bynnen betaleth (15, \& 1, Drittel 16, Jh.).

Stadtarchiv Lüneburg, AB 69: Einnahmen und Ausgaben sowie Rentenbriefe $(1534 / 1565,1566$, 1586)

Stadtarchiv Lüneburg, AB 71: Kopien von Verschreibungen des Rates aus der Zeit von 1566 bis 1616; $A B$ 80: Schuldbuch mit Ratsrenteverschreibungen.

\section{Sekundärliteratur}

Albers, Herrmann (1939): Die Anleihen der Stadt Bremen vom 14. bis zum 18. Jahrhundert, in: Veröffentlichungen aus dem Staatsarchiv der freien Hansestadt Bremen 3, S. 8-163.

Baum, Hans-Peter (1985): Annuities in Late Medieval Hanse Towns, in: The Business History Review 59, S. $24-48$.

Beyer, Otto (1901): Schuldwesen der Stadt Breslau im 14. und 15. Jahrhundert mit besonderer Berücksichtigung der Verschuldung durch Rentenverkauf, in: Zeitschrift des Vereins für Geschichte (und Alterthum) Schlesiens 35, S. 68-143.

Bohmbach, Jürgen (1969): Umfang und Struktur des Braunschweiger Rentenmarktes 1300-1350, in: Niedersächsisches Jahrbuch für Landesgeschichte 41, S. 119-133.

Bosch, Rudolf (2015): The Impact of Financial Crises on the Management of Urban Fiscal Systems and Public Debt. The Case of the Dutchy of Guelders, 1350-1550, in: Remi van Schaï (Hg.): Economies, Public Finances and the Impact of Institutional Changes in Interregional Perspective, Turnhout, S. 103-112.

Chilosi, David / Schulze, Max-Stephan / Volckart, Oliver (2018): Benefits of Empire? Capital market integration north and south of the Alps, 1350-1800, in: The Journal of Economic History 78, S. $637-672$.

Cremer, Otto (1937): Der Rentenkauf im mittelalterlichen Köln. Nach Schreinsurkunden des 12.14. Jahrhunderts (mit Urkundenanhang), Würzburg.

Dollinger, Philippe (1998): Die Hanse, 5. Aufl., Stuttgart.

Dürre, Hermann (1861): Geschichte der Stadt Braunschweig im Mittelalter, Braunschweig. 
Eberhardt, Ilse (1996): „Von des stades wegene utgegeven unde betalt“. Städtischer Alltag im Spiegel der Stadtrechnungen von Osnabrück, Osnabrück.

Fouquet, Gerhard (1989): Die Finanzierung von Krieg und Verteidigung in oberdeutschen Städten des späten Mittelalters (1400-1500), in: Bernhard Kirchgässner / Günter Scholz (Hg.): Stadt und Krieg, Sigmaringen, S. 41-82.

Fryde, Edmund B. / Fryde, Matthew M. (1963): Public Credit, with Special Reference to NorthWestern Europe, in: M. M. Postan / E. E. Rich / Edward Miller (Hg.): The Cambridge Economic History of Europe from the Decline of the Roman Empire, Bd. 3: Economic Organization in the Middle Ages, Cambridge, S. 430-553.

Fuhrmann, Bernd (2003): „Öffentliches“ Kreditwesen in deutschen Städten des 15. und 16. Jahrhunderts, in: Scripta Mercaturae 37, S. 1-17.

Fuhrmann, Bernd (2007): „Der rat aber war zu rat mer ewigs gelts zu verkauffen“- Das kommunale Kreditwesen Nürnbergs im 15. Jahrhundert, in: Harm von Seggern / Gerhard Fouquet / HansJörg Gilomen (Hg.): Städtische Finanzwirtschaft am Übergang vom Mittelalter zur Frühen Neuzeit, Frankfurt a. M., S. 139-167.

Fuhrmann, Bernd (2016): Rentenverkäufe der Stadt Nürnberg während des 15. und der ersten Hälfte des 16. Jahrhunderts, Hamburg.

Gilomen, Hans-Jörg (1984): Der Rentenkauf im Mittelalter, Habilitationsschrift, Basel, https:// www.hist.uzh.ch/dam/jcr:fffffff-e319-fie9-ffff-ffff92c8b4ad/Rentenkauf.pdf, letzter Zugriff am 12.01.2019.

Gilomen, Hans-Jörg (2003): Städtische Anleihen im Spätmittelalter. Leibrenten und Wiederkaufsrenten, in: Christian Hesse / Beate Immenhauser / Oliver Landolt / Barbara Studer (Hg.): Personen der Geschichte. Geschichte der Personen. Studien zur Kreuzzugs-, Sozial- und Bildungsgeschichte, Basel, S. 165-185.

Gilomen, Hans-Jörg (2007): Raum und Kommunikation, zwei Kategorien in der Erforschung des städtischen Haushaltswesens vom Spätmittelalter zur frühen Neuzeit, in: Harm von Seggern / Gerhard Fouquet / Hans-Jörg Gilomen (Hg.): Städtische Finanzwirtschaft am Übergang vom Mittelalter zur Frühen Neuzeit, Frankfurt a. M., S. 25-74.

Gilomen, Hans-Jörg (2018): Anleihen im Finanzhaushalt schweizerischer Reichsstädte insbesondere durch den Rentenverkauf, in: Michael Rothmann / Helge Wittmann (Hg.): Reichsstadt und Geld, Petersberg, S. 45-98.

Haemers, Jelle (2015): A Financial Revolution in Flanders? Public Debt, Representative Institutions, and Political Centralisation in the County of Flanders during the 1480s, in: Remi van Schaik (Hg.): Economies, Public Finances and the Impact of Institutional Changes in Interregional Perspective, Turnhout, S. 103-112.

Hauptmeyer, Carl-Hans (1997): Niedersächsische Wirtschafts- und Sozialgeschichte im hohen und späten Mittelalter (1000-1500), in: Ernst Schubert (Hg.): Geschichte Niedersachsens, Bd. 2, T. 1: Politik, Verfassung, Wirtschaft vom 9. bis zum ausgehenden 15. Jahrhundert, Hannover, S. 10411319.

Hefenbrock, Martin (1929): Lübecker Kapitalanlagen in Mecklenburg bis 140o, Dissertation, Kiel.

Hesse, Peter/Rothmann, Michael (2005): Zwischen Diplomatik und Diplomatie. Städtische Briefbücher als serielle Schlüsselzeugnisse städtischer Kommunikation im deutschen Spätmittelalter - Die Kölner Briefbücher von 1418 bis 1424. Ein Werkstattbericht, in: Geschichte in Köln 52, S. $69-128$.

Höhlbaum, Konstantin (Hg.) (1896): Kölner Inventar, Leipzig.

Huang, Angela / Chilosi, David / Sapoznik, Alexandra (2019): A Source Collection on Urban annuities, $14^{\text {th }}-18^{\text {th }}$ centuries. An Introduction to the Data, in: Vierteljahrschrift für Sozial- und Wirtschaftsgeschichte 106, S. 67-80.

Institut für historische Landesforschung der Universität Göttingen (Hg.): Niedersächsische Klosterkarte, http://www.landesgeschichte.uni-goettingen.de/kloester/, letzter Zugriff am 12.1.2019. 
Isenmann, Eberhard (2014): Die Deutsche Stadt im Mittelalter (1150-1550). Stadtgestalt, Recht, Verfassung, Stadtregiment, Kirche, Gesellschaft, Wirtschaft, Köln.

Knipping, Richard (1894): Das Schuldenwesen der Stadt Köln im 14. und 15. Jahrhundert, in: Westdeutsche Zeitschrift für Geschichte und Kunst 13, S. 340-394.

Koppmann, Karl (Hg.) (1869-1894): Kämmereirechnungen der Stadt Hamburg, 7 Bde., Hamburg.

Koppmann, Karl; u. a. (Hg.) (1870-1913): Die Rezesse und andere Akten der Hansetage, 3 Abt., Leipzig.

Lambert, Bart (2015): The Political Side of the Coin: Italian Bankers and the Fiscal Battle between Princes and Cities in the Late Medieval Low Countries, in: Remi van Schaïk (Hg.): Economies, Public Finances and the Impact of Institutional Changes in Interregional Perspective, Turnhout, S. $103-112$.

Lorenzen-Schmidt, Klaus-Joachim (1979/80): Der Kieler Rentenmarkt zwischen 1488 und 1560. Umfang und Dynamik des städtischen Kapitalmarktes unter Berücksichtigung der Rentenmärkte Krempes, Möllns und Hamburgs, in: Mitteilungen der Gesellschaft für Kieler Stadtgeschichte 67, S. 97-117.

Mack, Heinrich (1889): Die Finanzverwaltung der Stadt Braunschweig bis zum Jahre 1374, Breslau.

Moderhack, Richard (1985): Braunschweig. Das Bild der Stadt in 900 Jahren: Geschichte und Ansichten, T. 1: Braunschweigs Stadtgeschichte, Braunschweig.

Puhle, Matthias (1985): Die Politik der Stadt Braunschweig innerhalb des Sächsischen Städtebundes und der Hanse im späten Mittelalter, Braunschweig.

Rothmann, Michael (1998): Die Frankfurter Messen im Mittelalter, Stuttgart.

Rothmann, Michael (2007): Gemeiner Nutzen auf Kredit. Der Frankfurter Rentenmarkt und sein Einzugsgebiet im Spätmittelalter, in: Harm von Seggern / Gerhard Fouquet / Hans-Jörg Gilomen (Hg.): Städtische Finanzwirtschaft am Übergang vom Mittelalter zur Frühen Neuzeit, Frankfurt a. M., S. $183-238$.

Spiess, Werner (1966): Geschichte der Stadt Braunschweig im Nachmittelalter. Vom Ausgang des Mittelalters bis zum Ende der Stadtfreiheit (1491-1671), Braunschweig.

Steinführer, Henning (2017): Kurze Geschichte der Hansestadt Braunschweig, Braunschweig.

Zuijderduijn, Jaco (2009): Medieval Capital Markets. Markets for Renten, State Formation and Private Investment in Holland (1300-1550), Leiden.

DR. ANGELA HUANG

Forschungsstelle für die Geschichte der Hanse und des Ostseeraums, c/o Europäisches

Hansemuseum Lübeck, An der Untertrave 1, 23552 Lübeck, alhuang@fgho.eu

DR. ALEXANDRA SAPOZNIK

King's College London, Faculty of Arts \& Humanities, Department of History,

Strand, London WC2R 2LS, Great Britain, alexandra.sapoznik@kcl.ac.uk 